\title{
Electronic Structure Trends Across the Rare-Earth Series in Superconducting Infinite-Layer Nickelates
}

\author{
Emily Been, ${ }^{1,2}$ Wei-Sheng Lee, ${ }_{1}^{1}$ Harold Y. Hwang, ${ }^{1,3}$ Yi Cui, ${ }^{4}$ Jan Zaanen, ${ }^{5}$ \\ Thomas Devereaux $\odot,{ }^{1,4}$ Brian Moritz@ ${ }^{1}$ and Chunjing Jia ${ }^{1}$ \\ ${ }^{1}$ Stanford Institute for Materials and Energy Sciences, SLAC National Accelerator Laboratory, \\ 2575 Sand Hill Road, Menlo Park, California 94025, USA \\ ${ }^{2}$ Department of Physics, Stanford University, Stanford, California 94305, USA \\ ${ }^{3}$ Department of Applied Physics, Stanford University, Stanford, California 94305, USA \\ ${ }^{4}$ Department of Materials Science and Engineering, Stanford University, Stanford, California 94305, USA \\ ${ }^{5}$ Institute Lorentz for Theoretical Physics, Leiden University, 2300 RA Leiden, Netherlands
}

(Received 21 March 2020; revised 10 November 2020; accepted 24 December 2020; published 11 March 2021)

\begin{abstract}
The recent discovery of superconductivity in oxygen-reduced monovalent nickelates has raised a new platform for the study of unconventional superconductivity, with similarities to and differences from the cuprate high-temperature superconductors. In this paper, we investigate the family of infinite-layer nickelates $R \mathrm{NiO}_{2}$ with rare-earth $R$ spanning across the lanthanide series, introducing a new and nontrivial "knob" with which to tune nickelate superconductivity. When traversing from $\mathrm{La}$ to $\mathrm{Lu}$, the out-of-plane lattice constant decreases dramatically with an accompanying increase of $\mathrm{Ni} d_{x^{2}-y^{2}}$ bandwidth; however, surprisingly, the role of oxygen charge transfer diminishes. In contrast, the magnetic exchange grows across the lanthanides, which may be favorable to superconductivity. Moreover, compensation effects from the itinerant $5 d$ electrons present a closer analogy to Kondo lattices, indicating a stronger interplay between charge transfer, bandwidth renormalization, compensation, and magnetic exchange. We also obtain the microscopic Hamiltonian using the Wannier downfolding technique, which will provide the starting point for further many-body theoretical studies.
\end{abstract}

DOI: $10.1103 /$ PhysRevX.11.011050

Subject Areas: Condensed Matter Physics

\section{INTRODUCTION}

In 1986, the discovery of high-temperature superconductivity in the copper oxide (cuprate) $\mathrm{La}_{2-x} \mathrm{Sr}_{x} \mathrm{CuO}_{4}$ (LSCO) by Bednorz and Müller ushered in a new era in condensed matter research [1]. Soon after, a number of "families" of cuprates were discovered with electronic and structural similarities. While no consensus yet exists on the mechanism of superconductivity in these compounds, a number of characteristics seem to be common across the different families. Structurally, they consist of quasitwo-dimensional $\mathrm{CuO}_{2}$ planes separated by chargereservoir spacer layers, which may contain a number of different atoms, including both rare earth and/or oxygen. Electronically, the low-energy degrees of freedom primarily reside in the $\mathrm{CuO}_{2}$ planes, where formal valence counting would yield $\mathrm{Cu}^{2+}$ in a $3 d^{9}$ electronic configuration; and crystal-ligand-field effects would place the orbitals with

Published by the American Physical Society under the terms of the Creative Commons Attribution 4.0 International license. Further distribution of this work must maintain attribution to the author(s) and the published article's title, journal citation, and DOI. $d_{x^{2}-y^{2}}$ symmetry near the Fermi energy. Characterized as charge-transfer insulators within the Zaanen-SawatzkyAllen (ZSA) scheme [2], the undoped parent compounds are antiferromagnetic insulators due to strong correlations; and upon doping, one or more bands emerge and cross the Fermi level, depending on the material specifics. Aside from superconductivity, hole doping in the cuprates produces a rich, complex phase diagram with a number of distinct, and potentially intertwined, phases [3].

Research into unconventional superconductivitysuperconductivity that does not seem to fall within the BCS paradigm [4], as in the cuprates-has evolved over time, and the list of materials now includes heavy-fermion intermetallics, organic superconductors, ruthenates, and iron pnictides and chalcogenides. Although the cuprates share some characteristics with these compounds, especially the proximity of the superconducting state to antiferromagnetism [5], the lack of a material analog with both crystal and, more importantly, electronic structure similarities makes it more difficult to draw any general conclusions about a universal mechanism for unconventional superconductivity, if one exists at all.

Nickel oxide compounds, or nickelates, may provide such a platform, but they require some unconventional 
chemistry. In variants such as cubic $\mathrm{NiO}$ or single-layer $\mathrm{La}_{2} \mathrm{NiO}_{4}$, which is isostructural to the parent compound of the cuprate LSCO, the Ni-cation has a formal valence of $\mathrm{Ni}^{2+}$ in a nominal $3 d^{8}$ electronic configuration with spin $S=1$. These nickelates can be doped as the cuprates, for instance, with $\mathrm{Sr}$ or $\mathrm{O}$, but they remain insulating. Guided by theoretical predictions [6,7], this led to the original discovery of the electronic stripes, which turned out to be of the "filled" insulating type [8]. This finding, in turn, inspired the discovery of similar "spin stripes" in the LSCO family [9], with intriguing relations to charge order in other cuprate families [10]. A key difference was identified early on [7] in the form of much stronger electron-phonon interaction associated with the holes in the nickelates as compared to the cuprates, rooted in the strongly Ni-O hybridized lower Hubbard band [11], leading to much stronger static lattice deformations stabilizing the filled stripes $[7,8]$. Substantial literature emerged dedicated to the physics of these nickelate stripes (see, e.g., the very recent study [12]). Recent reports even suggest that a threelayer variant possesses similar stripe-ordered ground states [12].

Altering the chemical composition, $\mathrm{LaNiO}_{3}$ is a rareearth perovskite where the Ni-cation would have a formal valence of $\mathrm{Ni}^{3+}$ in a $3 d^{7}$ electronic configuration. However, this electronic configuration would be energetically unfavorable, such that this compound is instead a negative charge-transfer material with a nominal electronic configuration of $3 d^{8} \underline{L}$, where $\underline{L}$ stands for a ligand hole on oxygen [13]. Unfortunately, such materials also show no signs of superconductivity.

Using a chemical reduction process [14,15], the apical oxygens can be removed from the rare-earth perovskite nickelates, yielding rare-earth infinite-layer nickelates $R \mathrm{NiO}_{2}$, where $R$ can be any of a number of rare-earth atoms. These materials are isostructural to the infinite-layer cuprates with quasi-two-dimensional $\mathrm{NiO}_{2}$ planes separated by single layers of rare-earth atoms (see Fig. 1). Formal valence counting yields $\mathrm{Ni}^{1+}$-cations with a $3 d^{9}$ electronic configuration similar to the $\mathrm{Cu}^{2+}$-cations in the cuprates. The formal similarities with the cuprates do not stop there, as the recent discovery of superconductivity in ( $\mathrm{Nd}, \mathrm{Sr}) \mathrm{NiO}_{2}$, with a $T_{c}$ as high as $15 \mathrm{~K}$ [16], potentially makes these infinite-layer nickelates a new platform for studying unconventional superconductivity.

Previous theoretical work, based on density-functional theory (DFT) [17,18], and recent experiments [19] have highlighted similarities between the $R \mathrm{NiO}_{2}$ compounds, primarily with $R=\mathrm{La}$ or $\mathrm{Nd}$, and infinite-layer cuprates, as well as significant differences in their electronic structure. A host of new theoretical proposals [20-31], also primarily based on DFT, likewise find both similarities to and differences from the cuprates. While there is no overall consensus on the physics at this stage, there is more or less general agreement on a few observations about the

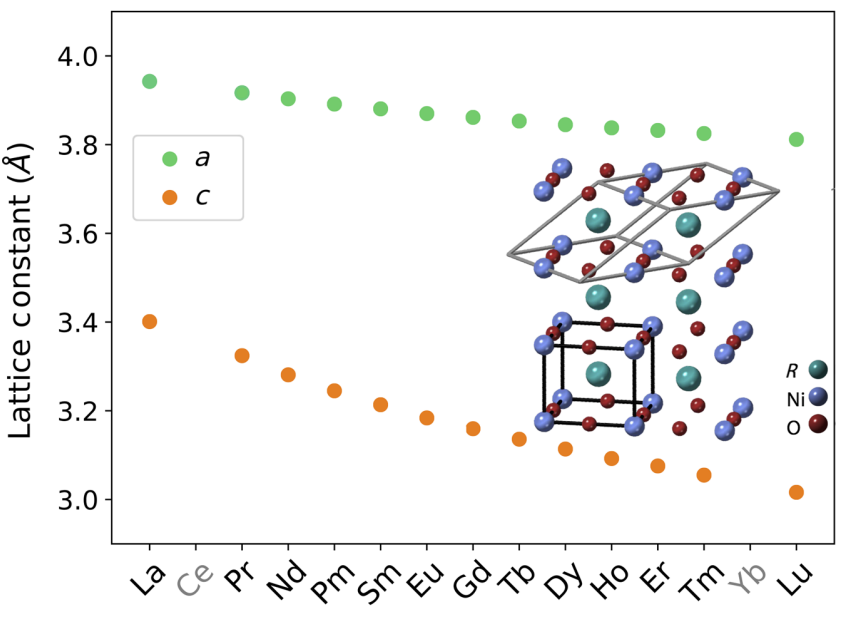

FIG. 1. Lattice constant and crystal structure of $R \mathrm{NiO}_{2}$. The figure shows the DFT(GGA) relaxed $R \mathrm{NiO}_{2}$ lattice constants. Inset: crystal structure for infinite-layer nickelate $R \mathrm{NiO}_{2}$. One-Ni unit cell (black) for the paramagnetic calculation and two-Ni unit cells (gray) for $(\pi, \pi, \pi)$ the antiferromagnetic calculation are shown, respectively.

electronic structure in $R \mathrm{NiO}_{2}$ : In the absence of strong interactions, the low-energy physics in the $\mathrm{NiO}_{2}$ layers is that of a single, holelike band crossing the Fermi energy; in the presence of strong interactions, the $\mathrm{NiO}_{2}$ layers become "Mott" insulating, although such a state still involves a significant amount of oxygen character [20]; and unlike the cuprates, rather than sitting well above the Fermi energy, the rare-earth band forms small, but significant, metallic pocket(s) $[18,19]$. Of course, this leaves much to explore. In particular, lacking any experimental signatures of magnetic order in the parent compounds [14,32], what is the nature of magnetism, if any, in $R \mathrm{NiO}_{2}$, and how might it evolve with changes in chemical composition and doping? What role does the metallic rare-earth band play, given its small size, and what about quasiparticle compensation and extinction? Ultimately, can one infer something universal about unconventional superconductivity from the rare-earth nickelates? Answering these questions, and many others, will require a great deal more experimental and theoretical work.

To at least begin probing for clues to the underlying physics in the rare-earth nickelates, and lifting the veil on general principles of unconventional superconductivity, here, we present a systematic investigation into the parent compound $R \mathrm{NiO}_{2}$, where $R$ runs across the entire lanthanide series. Based on our electronic structure calculations, we show that two bands near $E_{F}$ contribute to the physics of these materials. One quasi-two-dimensional band, comprised of Ni $3 d_{x^{2}-y^{2}}$ and in-plane $\mathrm{O} 2 p_{x}$ and $2 p_{y}$ orbitals, has a Zhang-Rice-singlet-like character but with a smaller oxygen contribution than in the cuprates, which decreases when going from $\mathrm{La}$ to $\mathrm{Lu}$. The bandwidth of this band increases, as does the in-plane magnetic exchange, also going from $\mathrm{La}$ to $\mathrm{Lu}$. The other band, comprised of 
rare-earth $5 d$ orbitals, crosses $E_{F}$ and forms a small, but significant metallic electron pocket. This $5 d$ band has a fully three-dimensional dispersion, corresponding to an itinerant electron picture with three-dimensional, longrange hoppings. These two bands hybridize, possibly forming a new type of Kondo- or Anderson-lattice system, where the strongly correlated $\mathrm{NiO}_{2}$ layer plays the role of the $4 f$ electrons in the heavy-fermion, rare-earth intermetallics. The electron density in the itinerant band is quite low, so it is unclear what effect it may have on the strongly correlated Ni $3 d_{x^{2}-y^{2}}$ band, which is more reminiscent of the low-energy electronic structure in the cuprates. Finally, we present a microscopic Hamiltonian and effective parameters for representative compounds, which can serve as a starting point for more complex many-body calculations for specific materials and the infinite-layer rare-earth nickelate family, in general.

\section{CRYSTAL STRUCTURE}

The parent compounds of the infinite-layer rare-earth nickelates $R \mathrm{NiO}_{2}$ have four atoms in a primitive unit cell with space group Pmmm: $\mathrm{Ni}$ at $(0,0,0), \mathrm{O}$ at $\left(\frac{1}{2}, 0,0\right)$ and $\left(0, \frac{1}{2}, 0\right)$, and $R$ at $\left(\frac{1}{2}, \frac{1}{2}, \frac{1}{2}\right)$-all in units of the lattice constants $a, b$, and $c$, respectively, with $a=b$, as shown schematically in the inset of Fig. 1. One expects that the size of the rare-earth atom decreases with increasing atomic mass $Z$. We optimize the lattice constants for compounds across the lanthanide series using DFT as implemented in Quantum ESPRESSO [33], with PAW pseudopotentials. As we will discuss in more detail, we choose to place the $4 f$ electrons in the core. As shown in Fig. 1, this optimization yields a systematic reduction of the out-of-plane lattice constant $c$ by about $10 \%$ across the lanthanide series, as well as a reduction of the in-plane lattice constant $a$ by about 5\%, assuming bulk material. Thin films grown on an $\mathrm{SrTiO}_{3}$ substrate (experimental in-plane lattice constant of about $3.91 \AA$ and GGA calculated in-plane lattice constant of about $1 \%$ larger [34]) for most of the lanthanide series would experience an in-plane tensile strain, which would tend to further reduce the out-of-plane lattice constant across the series.

Given the stacking, one expects that the out-of-plane lattice constant should reflect the decreasing radius of the trivalent rare-earth ions going to the right in the series, which actually matches the approximately $10 \%$ contraction of the lattice constants of the elemental rare-earth metals quite well [35]. However, this is in striking contrast with the $R \mathrm{Ba}_{2} \mathrm{Cu}_{3} \mathrm{O}_{7-\delta}$ system, where the changes in lattice constants are on the $1 \%$ level-accordingly, it was established early on that rare-earth substitution makes very little difference for the physical properties (including $T_{c}$ 's) in this cuprate family [36]. One may suspect that this considerable change of volume going through the series may have a more substantial relationship with the overall electronic structure in comparison to the 123 cuprates. This relationship is indeed the case according to our computations.

\section{ELECTRONIC STRUCTURE}

\section{A. General electronic structure features from DFT $+\boldsymbol{U}$ calculations}

The electronic structure of $R \mathrm{NiO}_{2}$ has been the subject of study for some time [17,18]. Generally, it has been recognized that the electronic structure near the Fermi energy consists of several features, including $\mathrm{Ni} 3 d$, O $2 p$, and $R 5 d[18,19]$. To investigate the evolution of these features with changes in the rare-earth element and to properly account for correlation effects in the quasi-twodimensional $\mathrm{NiO}_{2}$ plane, we perform density-functional mean-field calculations, including a Ni $3 d$ on-site Hubbard $U$, using the LDA $+U$ technique [37], which can provide information on general trends in the electronic structure across the lanthanide series.

Figure 2(a) illustrates the electronic structure of $\mathrm{NdNiO}_{2}$ along high-symmetry lines within the body-centered tetragonal (BCT) first Brillouin zone (BZ) for $U=0,3$, and $6 \mathrm{eV}$, respectively. The orbital character of the bands is superimposed on the electronic structure. Similar sequences for the other infinite-layer rare-earth nickelates, but without orbital character, appear in the Appendix A, Fig. 6. Although no explicit folding occurs for $U=0 \mathrm{eV}$, we plot the band structure in the two-Ni unit cell for ease of comparison. Starting with $U=0 \mathrm{eV}$, one observes four bands crossing the Fermi level: (1) two three-dimensional "Nd bands," forming pockets near the $\Gamma$ point; and (2) two quasi-two-dimensional "Ni bands." The two threedimensional pockets with predominantly Nd character form from the original pockets at the $\Gamma$ point and $A$ point in the unfolded tetragonal BZ. Note that only the $\mathrm{Nd}$ bands cross the Fermi energy along the $\Gamma-Z$ cut of Fig. 2(a). The two Ni bands have mixed $\mathrm{Ni}$ and $\mathrm{O}$ character. We will return to this point later. Note that there is a significant difference in the van Hove energy at the $X$ point between the original and folded bands (about a few hundred meV), highlighting the quasi-two-dimensional nature of these bands.

While the precise value for the Hubbard $U$ is not established, one can make reasonable guesses. The value would likely be larger than that of $\mathrm{Ni}$ metal $(\sim 2 \mathrm{eV})$ because of better screening of the interaction in the metal compared to $R \mathrm{NiO}_{2}$ compounds. Likewise, it would be smaller than the value in $\mathrm{NiO}(\sim 8 \mathrm{eV})$, a prototypical charge-transfer insulator in the ZSA scheme [2]. Figure 2(a) shows the band structure for $\mathrm{NdNiO}_{2}$ while systematically increasing the Hubbard $U$. First, the strong Coulomb repulsion forces the $\mathrm{Ni}$ bands to form characteristic upper and lower "Hubbard bands" separated by approximately $U$. As with the $\mathrm{O}$ character, we return to the ZSA classification later and simply refer to these as upper and lower Hubbard bands. These bands progressively renormalize and flatten with increasing Hubbard $U$. At the same time, the Nd pockets 

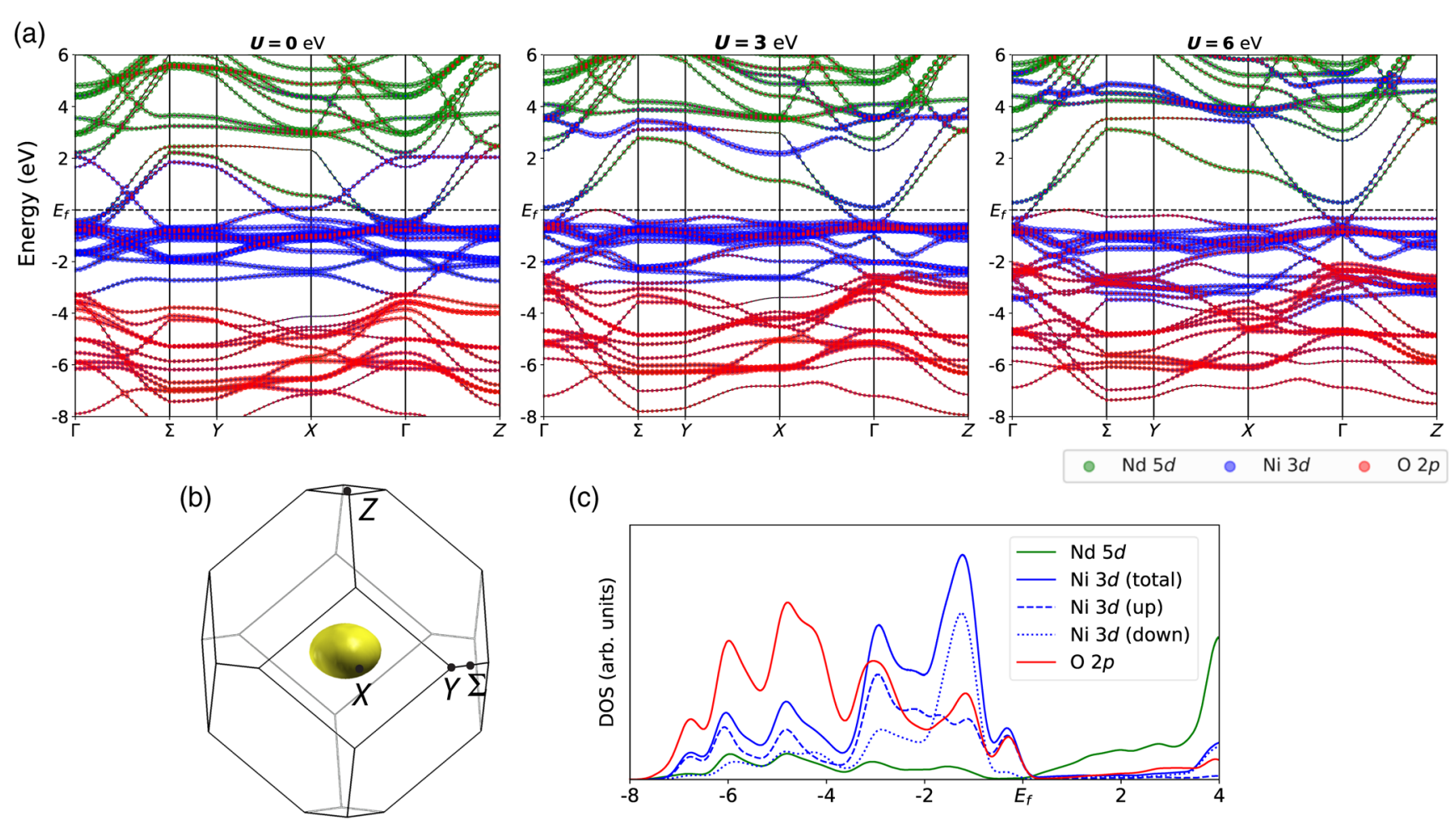

(c)

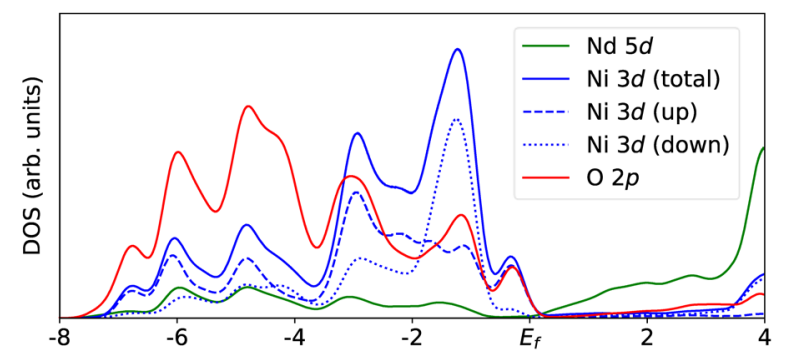

FIG. 2. Electronic structure of $\mathrm{NdNiO}_{2}$. (a) GGA and GGA $+U(U=3$ and $6 \mathrm{eV})$ calculations of the band structure and the corresponding atomic orbital character of $\mathrm{NdNiO}_{2}$. The "lower Hubbard band" and "upper Hubbard band" splitting in DFT $+\mathrm{U}$ scenarios are apparent; specifically, for $U=6 \mathrm{eV}$, they are located at about $-0.5-0 \mathrm{eV}$ and $3.5-5 \mathrm{eV}$ relative to $E_{f}$, respectively. (b) Visualization of Fermi surface geometry in the first BCT BZ for $\mathrm{NdNiO}_{2}$ in the GGA $+U(6 \mathrm{eV})$ calculation. (c) Partial density of states of $\mathrm{NdNiO}_{2}$ in $\mathrm{GGA}+U(6 \mathrm{eV})$ calculation-included are contributions from spin-polarized $\mathrm{Ni} 3 d$ states on one Ni atom to elucidate the symmetry in the two-Ni cell. In summary, the orbital character of the bands at the Fermi surface is dominated by Nd $5 d$, forming the electron pocket at the zone center. It is notable that this electron pocket becomes somewhat smaller but does not disappear with a larger value of $U$. In contrast, the nickel $3 d$ bands just below the Fermi energy hardly change with different $U$.

become progressively smaller, and one disappears entirely for $U=6 \mathrm{eV}$, with the band bottom just above the Fermi level at the $\Gamma$ point. The Fermi surface for $U=6 \mathrm{eV}$ consists of a single, electron pocket centered at the $\Gamma$ point with predominantly Nd character, as shown in Fig. 2(b).

Figure 2(c) shows the orbitally resolved partial density of states with $U=6 \mathrm{eV}$. First, note the small (albeit significant) Nd density of states at the Fermi energy, corresponding to the small Nd pocket in Fig. 2(d). Concentrating on $\mathrm{Ni}$ and $\mathrm{O}$, the $\mathrm{O} 2 p$ bands lie much further away from the Fermi energy than in other nickelates [19,38] or, more importantly, than in the cuprates [39], marking a system with significantly decreased oxidation level. This case is further confirmed by the mixing between $\mathrm{Ni}$ and $\mathrm{O}$ in the lower Hubbard band, which is much smaller compared to prototypical charge-transfer insulators like the cuprates. Specifically, the $\mathrm{O}$ content in the lower Hubbard band is much smaller in $\mathrm{RNiO}_{2}$ (between $40 \%$ and $48 \%$ ) than in $\mathrm{CaCuO}_{2}(\sim 75 \%)$ according to our DFT $+U$ calculations, as shown in Fig. 3(a) and Fig. 7 in Appendix B. These observations place $\mathrm{NdNiO}_{2}$ (and, more generally, $R \mathrm{NiO}_{2}$ ) well inside the Mott-Hubbard regime of the ZSA classification. As highlighted recently [20], as in the cuprates, one may view the doped holes as Zhang-Rice singletlike states, which are, however, now better defined because of their smaller O-like character. The results presented here are very much in line with such a picture, showing reduced $\mathrm{O}$ character in the lower Hubbard band, as well as recent $\mathrm{Ni}$ $L$-edge x-ray absorption (XAS) measurements, which confirm the $3 d^{9}$-like state of $\mathrm{Ni}$, and $\mathrm{O} K$-edge XAS, which highlights significant differences between the $\mathrm{O}$ character in infinite-layer nickelates when compared to other charge-transfer compounds, and the cuprates [19].

\section{B. Trends across lanthanide series: Bandwidth, oxygen content, and $R$ pocket}

A systematic survey across the lanthanides of the band structure with $U=0,3$ and $6 \mathrm{eV}$ and the density of states for the $U=6 \mathrm{eV}$ case is presented in the Appendix A, Fig. 6 . While variations exist with changes in the rare-earth element, Fig. 3 highlights some of the most important trends. For $U=0 \mathrm{eV}$ ("noninteracting" Ni bands), one can extract the effective bandwidth for the quasi-two-dimensional $\mathrm{Ni}$ bands, which includes not only the in-plane dispersion but also the contributions from any out-of-plane 
(a)

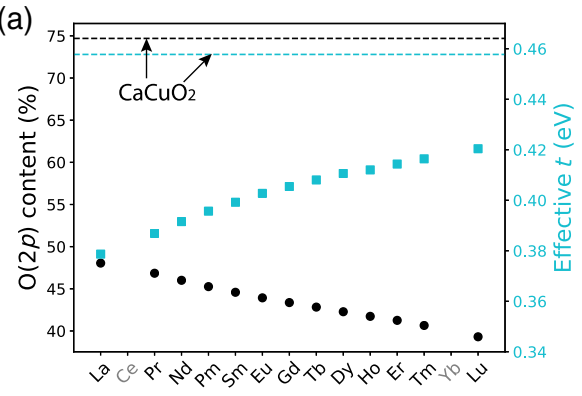

(b)

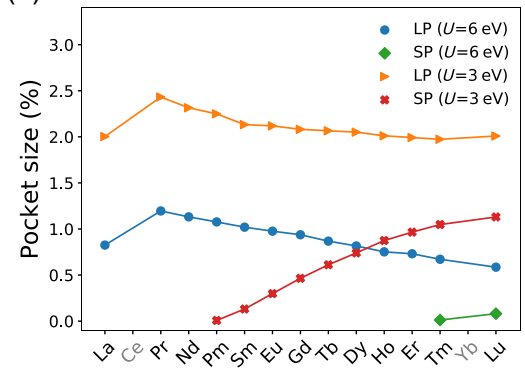

(c)
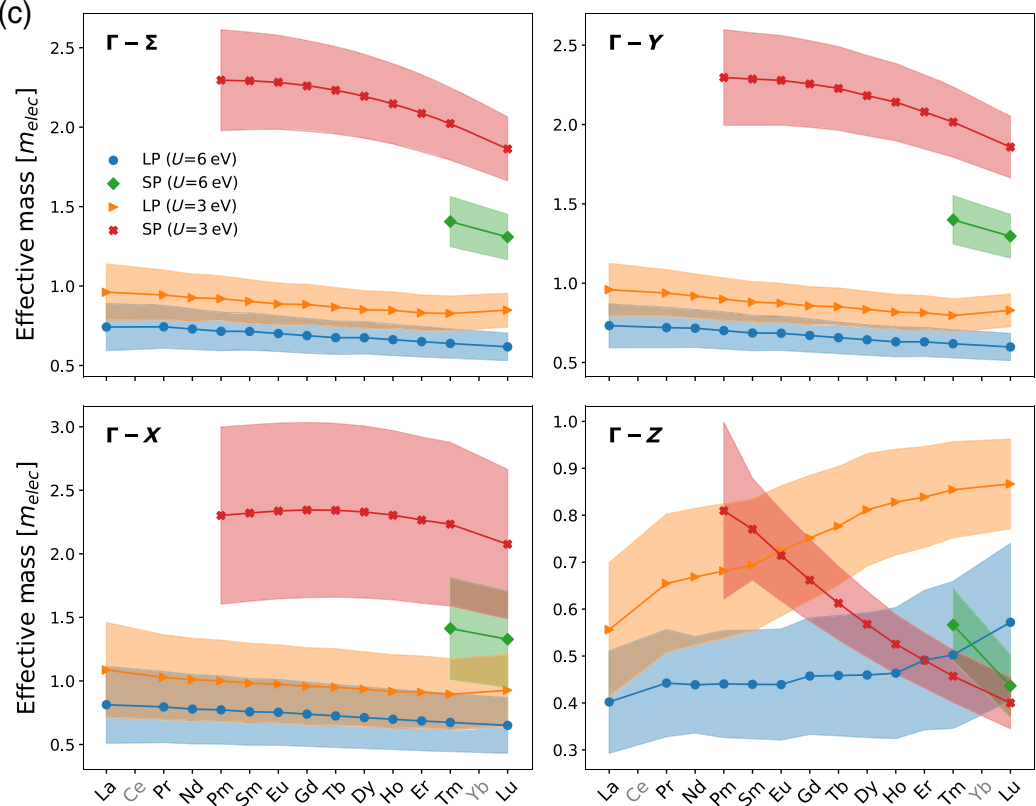

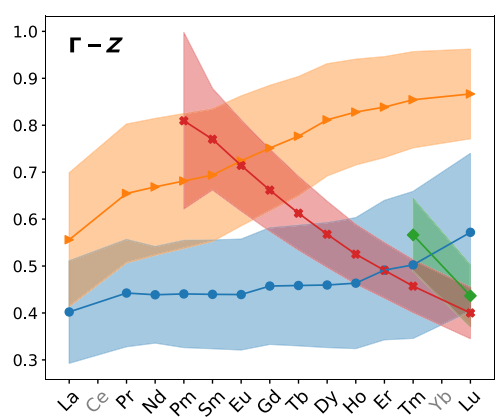

FIG. 3. Electronic structure of $R \mathrm{NiO}_{2}$. (a) Left axis: oxygen $2 p$ content in percentage of the total for the lower Hubbard model in the antiferromagnetic GGA $+U(U=6 \mathrm{eV})$ calculation for $R \mathrm{NiO}_{2}$ and $\mathrm{CaCuO}_{2}$. Right axis: effective $\mathrm{Ni} d_{x^{2}-y^{2}}$ nearest-neighboring hopping as estimated by $\frac{1}{8} \times$ bandwidth of the Ni $d_{x^{2}-y^{2}}$ band in the paramagnetic GGA calculation. The effective $\mathrm{Cu} d_{x^{2}-y^{2}}$ nearestneighboring hopping as estimated by $\frac{1}{8} \times$ bandwidth of the $\mathrm{Cu} d_{x^{2}-y^{2}}$ band in the paramagnetic GGA calculation is shown by the dashed line. (b) Rare-earth pocket size in percentage of the first BZ volume. A large pocket (LP) is present for all elements in the lanthanide series for both $U=3 \mathrm{eV}$ and $6 \mathrm{eV}$. A small pocket (SP) appears at Pm and the elements to the right for $U=3 \mathrm{eV}$. SP appears at Tm and the elements to the right for $U=6 \mathrm{eV}$. (c) Rare-earth electron pocket effective mass in all principal-axes directions. See Sec. VII for more information about how data processing was conducted.

hybridization. The trend appears in Fig. 3(a) (left axis). While the bandwidth is about $3 \mathrm{eV}$ for all compounds, it monotonically increases by about $10 \%$ across the lanthanides. This result should come as no surprise considering the trend in lattice constants shown in Fig. 1: A reduction in both the in-plane and out-of-plane lattice constants should lead to increased orbital overlap and, by extension, hybridization and bandwidth.

Let us now turn to the big discovery revealed by this band structure study. Given that the lattice is contracting while the bandwidth is increasing going toward the end of the series, one would expect that the degree of covalency associated with the holes in the Ni-O planes should also increase. However, this is not the case at all. The degree of oxygen admixture is easy to quantify from the band structure by inspecting the orbital content of the lower Hubbard band, and the result is shown in Fig. 3(a). This oxygen character is very large to the left of the series, as high as about $48 \%$ in La. But we observe that it decreases by as much as about $40 \%$ running across the lanthanide series. One expects such differences when one goes down in the periodic system, substituting, e.g., $\mathrm{O}$ for $\mathrm{S}$, but not with the substitution by different rare earth, famous for their chemical similarity. Figure 6 in the Appendix A may provide a clue regarding this apparent paradox. Observing the distribution of the $\mathrm{O}$ weight on a large energy scale, one can discern a trend: Running across the lanthanide series, the centroid of $\mathrm{O}$ weight monotonically moves to higher binding energy, by perhaps $1 \mathrm{eV}$ from La to $\mathrm{Lu}$. Such a change would lead to an increase in the charge-transfer energy and would offset any increases in hybridization relating to the mixing between $\mathrm{Ni}$ and $\mathrm{O}$. Given the unusual chemistry of these monovalent nickelates, the chemically "inert" lanthanides appear to exert a spectacular influence on the nature of the oxidation in the Ni-O perovskite planes.

Another discovery associated with the lanthanide substitution revolves around the evolution of the $R 5 d$ electron pockets. We already highlighted that, for $\mathrm{La}$, these pockets are sensitive to $U$. For $U=0 \mathrm{eV}$, GGA indicates the presence of "large" volume (LP) and "small" volume (SP) pockets. However, for $U=6 \mathrm{eV}$, the small pocket has disappeared, and only the LP remains, as indicated in Fig. 2(b). In Figs. 3(b) and 3(c), we characterize what happens with these pockets going through the series for $U=3$ and $6 \mathrm{eV}$. Focusing on the volume enclosed by the LP (orange and blue), we see from Fig. 3(b) that its volume is decreasing for increasing $U$, but the dependence of this volume on $R$ is weak. It is surprising that upon moving through the series, there is a critical point where the small pocket reemerges, which happens for $U=3 \mathrm{eV}$ at $\mathrm{Pm}$ while for $U=6 \mathrm{eV}$ one has to go all the way to the end of 
the series. Finally, we analyze the effective mass of these pockets at $k_{\mathrm{F}}$ along the high-symmetry directions in the BCTBZ[Fig. 3(c)]. We find that in the in-plane directions, not much happens upon changing $R$. All of the action is in the $c$ direction: The mass associated with the LP shows a significant increase in the series, while the SP shows a quite strong decrease upon going towards the end of the series.

Notably, these changes to the electronic structure cannot be explained by volume change alone. When the rare-earth $R$ is kept constant and the lattice parameters are changed by an amount comparable to those shown in Fig. 1, the second electron pocket never appears, small hole pockets start to appear along $\Gamma-\Sigma$ and $Y-X$, and the effective hopping $t$ is significantly lower.

\section{MICROSCOPIC HAMILTONIAN}

Based on the electronic structure discussed above, we propose a model for $R \mathrm{NiO}_{2}$, involving two-orbitals with an on-site Hubbard $U$ associated with the planar $\mathrm{NiO}_{2}$ band (Ni band):

$$
\begin{aligned}
H= & \sum_{k, \sigma}\left(\varepsilon_{k}^{R} n_{k, \sigma}^{R}+\varepsilon_{k}^{\mathrm{Ni}} n_{k, \sigma}^{\mathrm{Ni}}\right)+U \sum_{i} n_{i, \uparrow}^{\mathrm{Ni}} n_{i, \downarrow}^{\mathrm{Ni}} \\
& +\sum_{k, i, \sigma}\left(V_{k, i} c_{k, \sigma}^{\dagger} d_{i, \sigma}+\text { H.c. }\right),
\end{aligned}
$$

where the first term describes the noninteracting $R$ and $\mathrm{Ni}$ bands, the second term represents the on-site Hubbard interaction in the $\mathrm{Ni}$ band, and the last term describes the hybridization between the $\mathrm{Ni}$ and $R$ bands. Here, $\varepsilon_{k}^{\alpha}$ represents the momentum-resolved "noninteracting" band structure, $n_{k, \sigma}^{\alpha}$ is the usual number operator, and the hybridization term has been written to resemble the typical form for the Kondo- or Anderson-lattice model with a hybridization between the dispersive $R 5 d$ band, with $c_{k}\left(c_{k}^{\dagger}\right)$ operators, and the Hubbard-like Ni $3 d$ band, with $d_{i}\left(d_{i}^{\dagger}\right)$ operators.

We derive parameters for the microscopic Hamiltonian by performing Wannier downfolding on the band structure in the one-Ni unit cell with $U=0 \mathrm{eV}$. Figure 4(a) shows the band structure and the atomic orbital content of $\mathrm{NdNiO}_{2}$ from DFT calculations. The Ni $d_{x^{2}-y^{2}}$ band highlighted in blue crosses $E_{F}$. The other band crossing $E_{F}$ has mostly $\mathrm{Nd}$ $d_{3 z^{2}-r^{2}}$ character, with some $\mathrm{Nd} d_{x y}$ component close to the A point. Wannier downfolding for the two bands was then performed to obtain the microscopic Hamiltonian on the two maximally localized Wannier orbitals as implemented in Wannier90 [40], using atomic $\mathrm{Ni} d_{x^{2}-y^{2}}$ and $\mathrm{Nd} d_{3 z^{2}-r^{2}}$ as the initial configuration. The $\mathrm{Ni} d_{3 z^{2}-r^{2}}$ band was not included because its on-site energy is much lower than $\mathrm{Ni}$ $d_{x^{2}-y^{2}}$ [19], and although $\mathrm{Ni} d_{3 z^{2}-r^{2}}$ weight appears near $\mathrm{E}_{f}$, this comes primarily from the strong hybridization between Ni $d_{z^{2}}$ and $R d_{z^{2}}$. This weight will be incorporated naturally in the Wannier orbital centered on $R d_{z^{2}}$ when downfolded
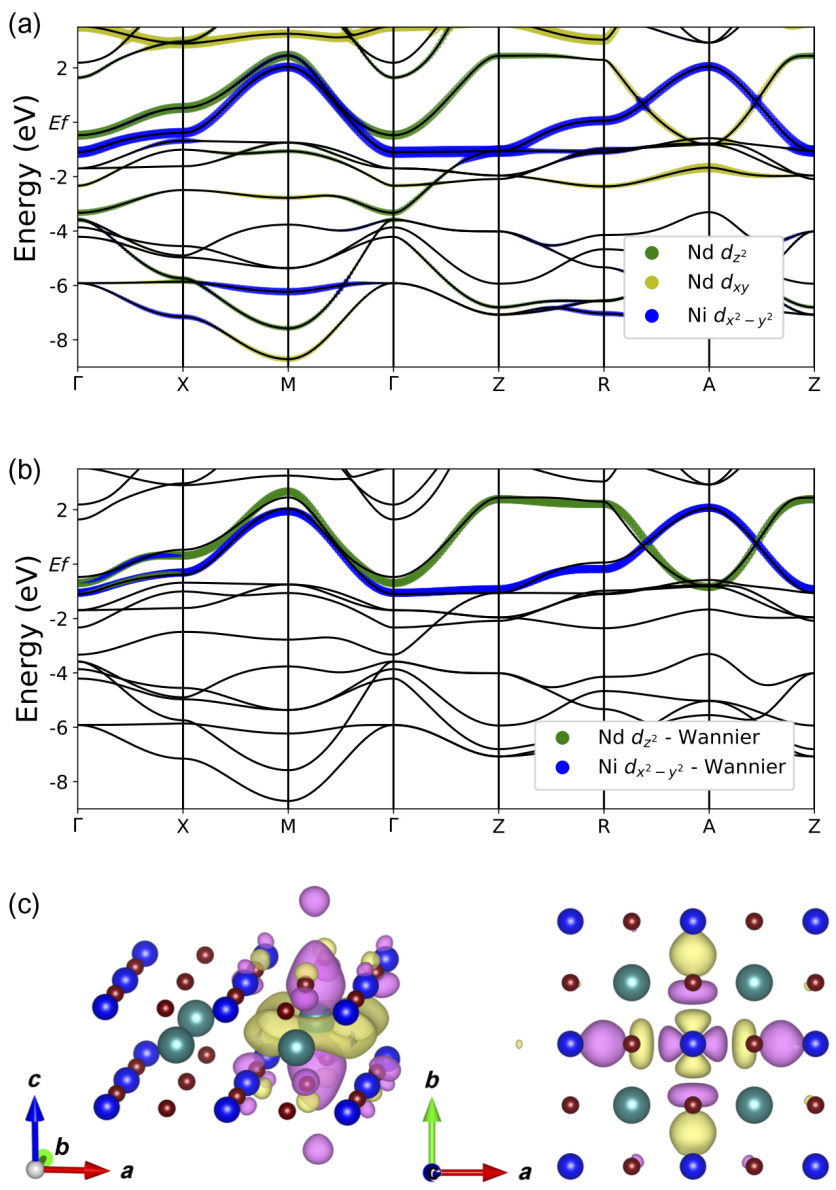

FIG. 4. Wannier downfolding of $\mathrm{NdNiO}_{2}$. (a) GGA calculation of $\mathrm{NdNiO}_{2}$ band structure, and $\mathrm{Nd} d_{z^{2}}$ (green), $\mathrm{Nd} d_{x y}$ (yellow), and Ni $d_{x^{2}-y^{2}}$ (blue) atomic orbital content. (b) Dispersion of twoorbital model based on the Wannier downfolding on the Wannier orbitals shown in panels (c). (c) $\mathrm{Nd} d_{z^{2}}$-like and Ni $d_{x^{2}-y^{2}}$-like Wannier orbitals.

to a two-orbital model. Further symmetrization to reserve the $D_{4 h}$ symmetry for the $\mathrm{Nd} d_{3 z^{2}-r^{2}}$ Wannier orbital and the corresponding Hamiltonian parameters are performed. The converged Wannier downfolding yields the dispersion shown in Fig. 4(b), with green highlighting the Nd character and blue highlighting the Ni character. The two bands mix significantly along $\Gamma-X$. The corresponding Wannier orbitals are shown in Fig. 4(c), where, by construction, the Nd Wannier orbital has $d_{3 z^{2}-r^{2}}$ symmetry centered on $\mathrm{Nd}$ and extends out of plane, with a rounded square shape in plane, while the Ni Wannier orbital has $d_{x^{2}-y^{2}}$ symmetry centered on $\mathrm{Ni}$ and extends onto the neighboring oxygen with $2 p$ symmetry. We have performed the Wannier downfolding approach on other $R \mathrm{NiO}_{2}$ materials for $R=\mathrm{La}, \mathrm{Pr}, \mathrm{Eu}$, and Dy, selectively. The parameters for these $R \mathrm{NiO}_{2}$ are shown in Table I. The table lists values above a minimum threshold of $10 \mathrm{meV}$, such that the noninteracting $R$ - and Ni-band dispersions take the form: 


$$
\begin{aligned}
\varepsilon_{k}^{R}= & \varepsilon_{0}^{R}+2 t_{[0,0,1]}^{R} \cos \left(k_{z}\right)+2 t_{[0,0,2]}^{R} \cos \left(2 k_{z}\right)+2 t_{[0,0,3]}^{R} \cos \left(3 k_{z}\right) \\
& +\left[2 t_{[1,0,0]}^{R}+4 t_{[1,0,1]}^{R} \cos \left(k_{z}\right)+4 t_{[1,0,2]}^{R} \cos \left(2 k_{z}\right)\right]\left[\cos \left(k_{x}\right)+\cos \left(k_{y}\right)\right] \\
& +\left[4 t_{[1,1,0]}^{R}+8 t_{[1,1,1]}^{R} \cos \left(k_{z}\right)+8 t_{[1,1,2]}^{R} \cos \left(2 k_{z}\right)+8 t_{[1,1,3]}^{R} \cos \left(3 k_{z}\right)\right] \cos \left(k_{x}\right) \cos \left(k_{y}\right) \\
& +\left[2 t_{[2,0,0]}^{R}+4 t_{[2,0,1]}^{R} \cos \left(k_{z}\right)\right]\left[\cos \left(2 k_{x}\right)+\cos \left(2 k_{y}\right)\right] \\
& +\left[4 t_{[2,1,0]}^{R}+8 t_{[2,1,1]}^{R} \cos \left(k_{z}\right)\right]\left[\cos \left(2 k_{x}\right) \cos \left(k_{y}\right)+\cos \left(k_{x}\right) \cos \left(2 k_{y}\right)\right], \\
\varepsilon_{k}^{\mathrm{Ni}}= & \varepsilon_{0}^{\mathrm{Ni}}+2 t_{[1,0,0]}^{\mathrm{Ni}}\left[\cos \left(k_{x}\right)+\cos \left(k_{y}\right)\right]+4 t_{[1,1,0]}^{\mathrm{Ni}} \cos \left(k_{x}\right) \cos \left(k_{y}\right)+2 t_{[2,0,0]}^{\mathrm{Ni}}\left[\cos \left(2 k_{x}\right)+\cos \left(2 k_{y}\right)\right] \\
+ & {\left[2 t_{[0,0,1]}^{\mathrm{Ni}}+4 t_{[1,0,1]}^{\mathrm{Ni}}\left[\cos \left(k_{x}\right)+\cos \left(k_{y}\right)\right]+8 t_{[1,1,1]}^{\mathrm{Ni}} \cos \left(k_{x}\right) \cos \left(k_{y}\right)\right] \cos \left(k_{z}\right) . }
\end{aligned}
$$

Recently, different model Hamiltonians have been proposed for the low-energy physics of infinite-layer nickelates [20-23], although our model Hamiltonian is

TABLE I. Hopping parameters for two-orbital Wannier down-

\begin{tabular}{|c|c|c|c|c|c|c|c|}
\hline \multicolumn{8}{|c|}{ Hopping parameters for Wannier downfolding } \\
\hline & & & $\mathrm{La}$ & $\operatorname{Pr}$ & $\mathrm{Nd}$ & $\mathrm{Eu}$ & Dy \\
\hline $\bar{i}$ & $\bar{j}$ & $k$ & \multicolumn{5}{|c|}{$t_{[i, j, k]}^{R}(\mathrm{eV})$} \\
\hline U & 0 & 0 & 1.132 & 1.280 & 1.305 & 1.360 & 1.413 \\
\hline & 0 & 1 & -0.022 & -0.132 & -0.164 & -0.214 & -0.240 \\
\hline 0 & 0 & 2 & -0.112 & -0.153 & -0.172 & -0.185 & -0.223 \\
\hline & 0 & 3 & 0.019 & & & & \\
\hline 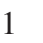 & 0 & 0 & -0.028 & -0.025 & -0.028 & -0.033 & -0.016 \\
\hline 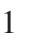 & 0 & 1 & -0.164 & -0.214 & -0.206 & -0.209 & -0.220 \\
\hline 1 & 0 & 2 & 0.033 & 5 & 0.030 & 0.036 & 0.043 \\
\hline & 1 & 0 & -0.062 & -0.079 & -0.090 & -0.061 & -0.082 \\
\hline & 1 & 1 & 0.024 & 0.060 & 0.066 & 0.052 & 0.068 \\
\hline 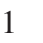 & 1 & 2 & 0.013 & 0.019 & 0.021 & 0.017 & 0.011 \\
\hline 1 & 1 & 3 & -0.019 & & & & \\
\hline 2 & 0 & 0 & & 6 & & 23 & 0.0 \\
\hline 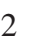 & 0 & 1 & 0.009 & -0.007 & -0.012 & -0.009 & -0.010 \\
\hline 2 & 1 & 0 & & -0.014 & -0.021 & -0.020 & -0.023 \\
\hline 2 & 1 & 1 & 0.009 & & & & \\
\hline 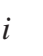 & $j$ & $k$ & \multicolumn{5}{|c|}{$t_{[i, j, k]}^{\mathrm{Ni}}(\mathrm{eV})$} \\
\hline 0 & 0 & 0 & 0. & 0.312 & 0.308 & 0.314 & 0.319 \\
\hline 1 & 0 & 0 & -0.355 & -0.376 & -0.374 & -0.370 & -0.385 \\
\hline 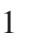 & 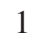 & 0 & & & 0.094 & 0.072 & 0.095 \\
\hline 2 & 0 & 0 & -0.043 & -0.033 & -0.043 & -0.036 & -0.0 \\
\hline 0 & 0 & 1 & -0.043 & -0.038 & -0.033 & -0.044 & -0.061 \\
\hline 1 & 1 & 1 & 0.013 & 0.021 & & 0.026 & \\
\hline 1 & 0 & 1 & & & & -0.020 & \\
\hline 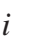 & $j$ & $k$ & \multicolumn{5}{|c|}{$t_{[i, j, k]}^{R-\mathrm{Ni}}(\mathrm{eV})$} \\
\hline 2 & 0 & 0 & -0.026 & 0.021 & 0.020 & 0.017 & 0.016 \\
\hline 2 & 0 & 2 & 0.013 & & & & \\
\hline
\end{tabular}
folding of $R \mathrm{NiO}_{2}(R=\mathrm{La}, \mathrm{Pr}, \mathrm{Nd}, \mathrm{Eu}$, and Dy). The hopping terms within $\mathrm{Ni}$ or $R$ bands represent the matrix elements as shown in Eqs. (2) and (3). The cross terms represent the matrix elements for $\sum_{i_{0}, j_{0}, k_{0}, i, j, k} t_{[i, j, k]}^{R-\mathrm{Ni}} c_{i_{0}, j_{0}, k_{0}}^{\dagger R} c_{i_{0}+i, j_{0}+j, k_{0}+k}^{\mathrm{Ni}}+$ symmetrically equivalent terms + H.c. The fractional coordinates are $R=$ $[0.5,0.5,0.5]$ and $\mathrm{Ni}=[0,0,0]$ in the paramagnetic unit cell. functionally unique. Including $\mathrm{Ni} d_{x^{2}-y^{2}}$ and $R d_{z^{2}}$ is universal as they capture the main features of the bands across $E_{F}$, according to both paramagnetic DFT calculations in single-Ni unit cells and antiferromagnetic DFT $(+U)$ calculations in two-Ni unit cells. Some theories take a multiorbital model (more than two orbitals), including even Ni $d_{z^{2}}$ or $R d_{x y}$, into consideration. We note that our two-orbital downfolded model can capture the contributions from $R d_{x y}$ and $\mathrm{Ni} d_{z^{2}}$ as well. Our $R d_{z^{2}}$-like Wannier orbital has a non-negligible contribution on the $\mathrm{Ni}$ $d_{z^{2}}$ character, as shown in Fig. 4(c), naturally capturing this $\mathrm{Ni} d_{z^{2}}$ contribution. Moreover, based on the DFT $+U$ band structure, the Ni $d_{z^{2}}$ contribution only appears at the bottom of the upper valence band at the $X$ point, making it less relevant in the case of light hole doping. Our downfolded $R$ $d_{z^{2}}$-like Wannier orbital, as shown in Fig. 4(c), has lower symmetry than the atomic $d_{z^{2}}$ orbital, capturing some contribution from the $d_{x y}$ orbital. Our two-orbital model, as shown in Fig. 4, also captures well the dispersion around the $A$ point, where the $R d_{x y}$ pocket is present. For these reasons, we believe that our proposed two-orbital model is the simplest model capturing the correct orbital contribution for infinite-layer nickelates.

\section{MAGNETISM AND SUPEREXCHANGE}

Regardless of one's specific beliefs, there is a general consensus that magnetism is a key aspect underlying the physics of the cuprates [41-44]. As already mentioned, so far, there are no experimental results that shed light on the magnetism of the infinite-layer rare-earth nickelates [16], although this continues to be the subject of aggressive experimental inquiry. Nevertheless, our density-functional theory calculations using LDA $+U$ possess (and our simple effective model likely possesses) an antiferromagnetic ground state. Recent articles have attempted to address the subject of superexchange and magnetism, specifically for $\mathrm{NdNiO}_{2}$ [21-23]. Some theories highlight similarities to the cuprates [21], while others report substantive differences, ranging from a nonmonotonic doping dependence of the magnetism [18] to an order-of-magnitude reduction in the effective superexchange [22]. 
Our effective model Eq. (1), as based on first principles, bares resemblance to a Kondo- or Anderson-lattice model. Well known from the heavy-fermion intermetallics, the Kondo coupling between the itinerant valence electrons and the localized $f$ electrons or spins may lead to magnetism and magnetic behavior driven by the Ruderman-KittelKasuya-Yosida interaction [45-47], in turn competing with the tendency to form nonmagnetic Kondo singlets [48]. Compared to these mainstream systems, two factors in the electronic structure of the $R \mathrm{NiO}_{2}$ series set it apart: (1) the relatively small size of the rare-earth pocket and (2) the single-band Hubbard-like nature of the electronic structure in the $\mathrm{NiO}_{2}$ layers.

The modest pocket size implies that the Kondo screening should be limited, given the fact that there is only a fraction of an itinerant electron available per localized spin (Noziere's exhaustion principle [49,50]). This "Kondo physics" may be quite sensitive to the details of these pockets, and one may anticipate that it will therefore be sensitive to the rare-earth substitution [see Fig. 3(b)].

The other novelty is the large direct hybridization between the $\mathrm{Ni} d^{9}$ states. This hybridization will lead to strong superexchange interactions between the $\mathrm{Ni}$ spins, which we predict to be large compared to the Kondo couplings involving the electron pockets. For this reason, one may better view the situation as being closely related to the effective single-band Hubbard models used to describe the low-energy physics in the cuprates, enriched by corrections coming from the small-pocket Kondo physics. Given the separation of scales, the consequences of these "Kondo corrections" on the physics of (doped) Mott insulators should be tractable to quite a degree using perturbation theory, but this particular situation appears to be completely unexplored at present.

Let us quantify these superexchange couplings. Considering either the effective bandwidth [Fig. 3(a)] or the Wannier downfolding (Table I), the kinetic scale $t_{\text {eff }}$ in the $R \mathrm{NiO}_{2}$ series is very similar to that in the cuprates. The nature of the effective interaction provides a distinction. The cuprate parent compounds are charge-transfer insulators with $U_{\text {eff }} \sim \Delta \sim 3 \mathrm{eV}$. The $R \mathrm{NiO}_{2}$ series features a charge transfer $\Delta \sim U$ and a reduced $\mathrm{O}$ content in the lower Hubbard band, placing these compounds closer to the single-band Mott insulators, at least within the $\mathrm{NiO}_{2}$ layer, such that $U_{\text {eff }} \sim U \sim 6 \mathrm{eV}$. Taking the standard perturbative expression $J=4 t_{\text {eff }}^{2} / U_{\text {eff }}$ for the superexchange, the value in the $\mathrm{NiO}_{2}$ layer would be smaller than that in cuprates but only by approximately a factor of 2 , or even less. To further quantify this comparison, we show the perturbative value of the superexchange $J$ across the lanthanide series in Fig. 5. We use both the Wannierdownfolded nearest-neighbor hopping $\left(t_{[1,0,0]}^{\mathrm{Ni}}\right.$ from Table I) and one-eighth of the in-plane bandwidth to estimate $t_{\text {eff }}$. The later slightly overestimates the effective hopping due to longer-range terms. To verify that using a static value of $U$ is

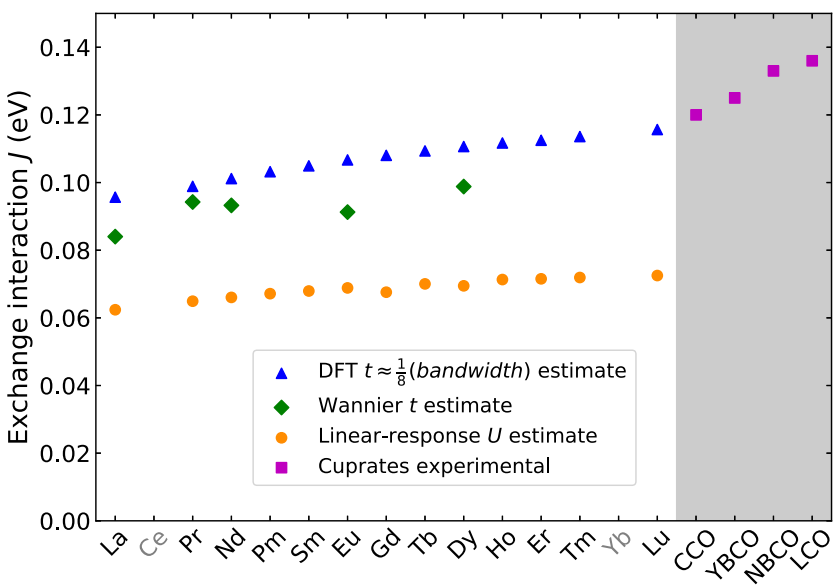

FIG. 5. Exchange interaction of $\mathrm{RNiO}_{2}$. Blue triangles show the exchange interaction $J$ calculated by $4 t^{2} / U$, where $t$ is estimated by $\frac{1}{8} \times$ bandwidth of the Ni $3 d_{x^{2}-y^{2}}$ band. Green diamonds show the exchange interaction $J$ calculated by $4 t^{2} / U$, where $t$ is the Wannier downfolding nearest-neighbor hopping for the $\mathrm{Ni}$ $3 d_{x^{2}-y^{2}}$ band. Orange circles are the exchange interaction $J$ calculated by $4 t^{2} / U$, where $t$ is estimated by $\frac{1}{8} \times$ bandwidth of the Ni $3 d_{x^{2}-y^{2}}$ band and $U$ is calculated by the linear-response method [51]. The cuprate exchange interactions shown by magenta squares are taken from Ref. [52-55].

a reasonable approximation in the estimate of $J$ across the lanthanides, we use a DFT perturbation linear-response method to calculate $U_{\text {lr }}$ for each $R \mathrm{NiO}_{2}$ [51]. This calculation gives a higher average value of $U_{\mathrm{lr}}=9.36 \mathrm{eV}$ but with a very small standard deviation of only $0.15 \mathrm{eV}$. As one can see from Fig. 5, a clear trend emerges across the lanthanide series in each case, with $J$ getting progressively larger. For comparison, we show experimental estimates of $J$ in the cuprates for $\mathrm{CaCuO}_{2}$ [55], $\mathrm{La}_{2} \mathrm{CuO}_{4}$ [53], $\mathrm{HgBCO}$ [54], and NBCO [52]. The conclusion is that the magnitude and nature of the superexchange interactions in the nickelate are predicted to be similar to the spin-spin interactions in the cuprates.

\section{OUTLOOK}

One of the dimensions that can be used in the laboratory to find out which factors matter for electronic properties, like superconductivity, is chemical composition. In the case of the present monovalent nickelates, preparing samples takes much effort, to the degree that high-quality bulk samples are not yet available. Band-structure methods have evolved in such a way that they are trustworthy with regard to predicting the large numbers of relevance to the microscopic nature of the electron systems. In this spirit, we explored the role of the rare-earth ions in the monovalent nickelates: Is it worthwhile to substitute various rareearth ions?

Given their chemical similarity, we anticipate that such substitutions are rather easy to accomplish. However, it 
comes at a price: Given the chemical similarity of the lanthanides, not much may change in the electronic structure. The case in point is in the early history of the cuprates, where it was discovered that the rare-earth substitution had a disappointing effect on the superconducting $T_{c}$ 's in the YBCO system [36]. But the monovalent nickelates are predicted to be, in this regard, quite different from the cuprates. In the latter, the ionic spacer layers may be viewed as lanthanide oxides; the lanthanide ions reside in caches formed from oxygen, and upon changing the ionic radii of the former, the oxygen packing has the effect that barely anything happens with the lattice constants. The monovalent nickelates are special in that all of these oxygens are removed and the Ni-O perovskite layers are kept apart by just the lanthanide ions. As we showed, the outcome is that, in particular, the $c$-axis lattice constant is shrinking substantially going from left to right in the series, by a magnitude similar to that found in the elemental rareearth metals.

According to our calculations, substituting rare earths in this nickelate is an interesting "knob" to tune microscopic parameters governing the electron system. Given the knowledge accumulated in the cuprates, there is reason to suspect that these may well be important for matters like the superconducting transition temperature. However, in the cuprates, they cannot be as neatly controlled as in the nickelates, given that there is no analogy of the "lanthanide volume control knob" that we identified in the nickelates.

We find that the gross picture for the electronic structure of the monovalent nickelates is closely related to the cuprates. It consists of nearly decoupled two-dimensional Hubbard-like systems living on a square lattice. Without doping, they would form a Mott-insulating, unfrustrated, Heisenberg antiferromagnet, becoming a "Mottness" metal upon doping, were it not for the fact that these layers are immersed in a low-density three-dimensional Fermi-liquid formed from rare-earth $5 d$-like conduction-band states. The latter are characterized by small electronlike Fermi pockets rendering the system metallic even at half-filling, while these metallic electrons are subjected to a subdominant Kondo-like interaction with the strongly correlated Ni-O $d_{x^{2}-y^{2}}$ electrons.

How can the "lanthanide knob" be exploited to find out how this electron system works? A first expectation is that the 3D "pockets" may be altered by the shrinking $c$ axis. However, we find that the influence of the $c$ axis on the pockets is rather marginal: The LP actually shrinks, while another small pocket may spring into existence pending the (unknown) value of $U$. Quite generally, one may turn it around to claim that measurement of this pocket, e.g., by photoemission or even quantum oscillations, may be quite informative regarding the details of the microscopic electronic structure that cannot be determined accurately on theoretical grounds (like the $U$ ).

Since the very beginning [39], a debate has been raging in the high- $T_{c}$ community that continues to this day [56] regarding whether one can get away with a single-band Hubbard model or whether it is crucial to take into account the multiband nature of the electronic structure, with prime suspects being the $\mathrm{O} 2 p$ states. A complication is that the way in which the hole states are distributed over oxygen and copper in the various cuprate families is a rather complex affair, resting on chemistry that is not easy to control systematically. The gravity of this affair is highlighted in a recent study exploiting nuclear quadrupole resonance (NQR) to determine these charge distributions [57,58], which shows a rather strong correlation between the magnitude of the $T_{c}$ 's and the hole density on oxygen throughout the landscape of cuprate families. In this regard, the monovalent nickelates may be a new arena in which to study the issue. Is it as simple as $T_{c}$ 's being lower because the oxygen band is at a higher chargetransfer energy than in the cuprates, with the effect that these materials are closer to a "single-band" picture? The main discovery of our study is the unanticipated strong dependence of the oxygen character of the holes as a function of the lanthanide, highlighted in Fig. 3(a). As we already stressed, lanthanide substitution should be easy to accomplish with the main effect, according to our computations, being to substantially change the oxygen character of the holes. This oxygen content is, in principle, measurable (e.g., by NQR, core hole spectroscopies). Assuming that there is, indeed, a strong correlation between the oxygen character and $T_{c}$ [57], the prediction follows that the optimal $T_{c}$ should decrease strongly upon substituting heavier lanthanides, given that, according to our results, the other microscopic electronic structure parameters should change only marginally. Such a possibility awaits rigorous experimental trial.

\section{METHODS}

Density-functional theory GGA and GGA $+U$ calculations for $R \mathrm{NiO}_{2}$ were performed using the GGA method and the simplified version by Cococcioni and de Gironcoli [59], as implemented in Quantum ESPRESSO [33], employing PAW pseudopotentials with the $4 f$ electrons placed in the core (except for La), explicitly to cope with the inability of current DFT functionals to properly treat the localized $4 f$ electrons. Ytterbium was not included in this rare-earth series analysis because the available pseudopotentials force zero occupation of the $5 d$ orbitals, resulting in fewer total valence electrons treated explicitly in the DFT calculations. This difference led to drastic changes in the $\mathrm{Yb}$ band structure from the trends observed over the rest of the series. Cerium was not included in this rare-earth series analysis because of the possible mixed valency. The remaining rare-earth elements had identical pseudopotential valence configurations.

The self-consistent field (SCF) calculations used a k-point Monkhorst-Pack grid of $16 \times 16 \times 16$; the nonself-consistent field (NSCF) calculation was run 
with 1000 evenly distributed k-points for PDOS. In the band-structure calculations, there were $20 \mathrm{k}$-points along all high-symmetry lines, except along $\Sigma-Y$, which was 10. For a better estimate of pocket size, in the PDOS calculations, a Gaussian broadening of $0.340 \mathrm{eV}$ (0.025 Ry) and an energy grid step of $0.0025 \mathrm{eV}$ were used.

In the two-Ni BCT unit cell and corresponding BZ, we found that an antiferromagnetic solution with wave vector $(\pi, \pi, \pi)$ leads to the lowest energy. For each rare-earth element, the $R \mathrm{NiO}_{2}$ crystal structure was relaxed with an unrestricted variable cell relaxation as implemented in Quantum ESPRESSO. These relaxations were done with the Hubbard $U$ turned off and no spin polarization. All band structures were calculated using each rare-earth element's relaxed crystal structure.

GGA $+U$ calculations for $\mathrm{CaCuO}_{2}$ were performed using the Vienna $A b$ initio Simulation Package (VASP) [60]. Including a Hubbard $U$ leads to spin polarization with the lowest-energy states supporting a $G$-type antiferromagnet $[(\pi, \pi, \pi)$ spin ordering $]$, slightly more energetically favorable than other types of antiferromagnetism or ferromagnetic ordering. Antiferromagnetism doubles the unit cell (as shown in the inset of Fig. 1) and folds the bands within a new BCT BZ. The DFT $+U$ calculations shown in this study were all performed with antiferromagnetic calculations with wave vector $(\pi, \pi, \pi)$ in two-Cu BCT.

As already announced, we placed the $4 f$ electrons in the core. Especially for DFT $+U$ calculations, this process saves quite some computational effort, with the added benefit that the figures representing the band structure do not get obscured by the many $4 f$ bands, which likely are irrelevant for our purposes. Such a practice is usually legitimate when dealing with rare-earth ions in ionic salts. The controlling factor is whether the $R$ ions have a "stable" valency. For instance, the trivalent $\mathrm{Ce}$ ion is characterized by a $4 f^{1}$ occupancy of the $f$ shell. Given that the $f$ shell is "hidden" in the core of the ion, it is characterized by a very large $U>10 \mathrm{eV}$, while the hybridization with the valence states is minute. The outcome is that this $f$ electron turns into a strongly localized $4 f$ spin that barely interacts with the other electrons. In most cuprates, one finds that this rare-earth spin system does not interfere with superconductivity and other intertwined orders at typical transition temperatures, while it shows a transition to an ordered magnetic state at very low temperatures driven by dipolar interactions. These $4 f$ states only become active when dealing with a mixed valent situation. The tetravalent state may become energetically close to the trivalent state, implying, for example, that in $\mathrm{Ce}$, one encounters a $4 f^{1}-$ $4 f^{0}$ valence fluctuation. This requires fine-tuning but cannot be excluded beforehand. In the cuprate context, a famous example is the $\mathrm{PrBa}_{2} \mathrm{Cu}_{3} \mathrm{O}_{6.5}$ system, which turns into an antiferromagnetic insulator due to the mixed valence of the $\operatorname{Pr}$ ion [61].

The oxygen ratio in total DOS was determined by integrating the projected density of states (PDOS) corresponding to the lower Hubbard band for $U=6 \mathrm{eV}$. The PDOS used for this integration had a Gaussian broadening of $0.054 \mathrm{eV}(0.004 \mathrm{Ry})$, much lower than those shown in either Fig. 3 or the Appendix A, Fig. 6. The integration used the trapezoidal method as implemented in PYTHON 3's numpy.trapz $[62,63]$. The range of integration was chosen by taking zeros of the first derivative of the PDOS near the Fermi energy and near the first local minimum (about $-0.5 \mathrm{eV}$ ), which effectively straddles the lower Hubbard band.

The size of the rare-earth electron pocket, or the Fermi surface volume as shown in Fig. 3(b), was determined by approximating the surface as an ellipsoid: The first two principle semiaxes approximate a circle in plane, whose lengths are given by the average distance along the $\Gamma-\Sigma$, $\Gamma-Y$, and $\Gamma-X$ directions; the length of the third principal semiaxis comes from the distance along $\Gamma-Z$. We report the volume as a percentage of the total volume of the first BZ. A higher-resolution k-point path near $\Gamma$ was used in determining the Fermi energy crossings $k_{\mathrm{F}}$ for the rare-earth bands along these high-symmetry cuts, with 200 k-points in each direction.

Using the same higher-resolution data near $\Gamma$, the effective mass was calculated from the second derivative of a parabolic fit to the band structure at the Fermi energy $k_{\mathrm{F}}$. For parabolic fitting, ten points along the band both above and below $k_{\mathrm{F}}$ were chosen and then mirrored symmetrically about $\Gamma$ (40 points total per fit). These points were fit with a second-order polynomial $a x^{2}+b x+$ $c$ [64], such that $d^{2} E / d k^{2}=a \pm \delta a$, where $\delta a$ is the standard deviation of the fit parameter.

\section{ACKNOWLEDGMENTS}

We thank Danfeng Li, Hye-ok Yoon, and Das Pemmaraju for insightful discussions. The work at Stanford University and SLAC National Accelerator Laboratory was supported by the U.S. Department of Energy, Office of Basic Energy Sciences, Division of Materials Sciences and Engineering, under Contract No. DE-AC02-76SF00515. This research used resources of the National Energy Research Scientific Computing Center (NERSC), a U.S. Department of Energy Office of Science User Facility, operated under Contract No. DEAC02-05CH11231. 


\section{APPENDIX A: LANTHANIDE SERIES}
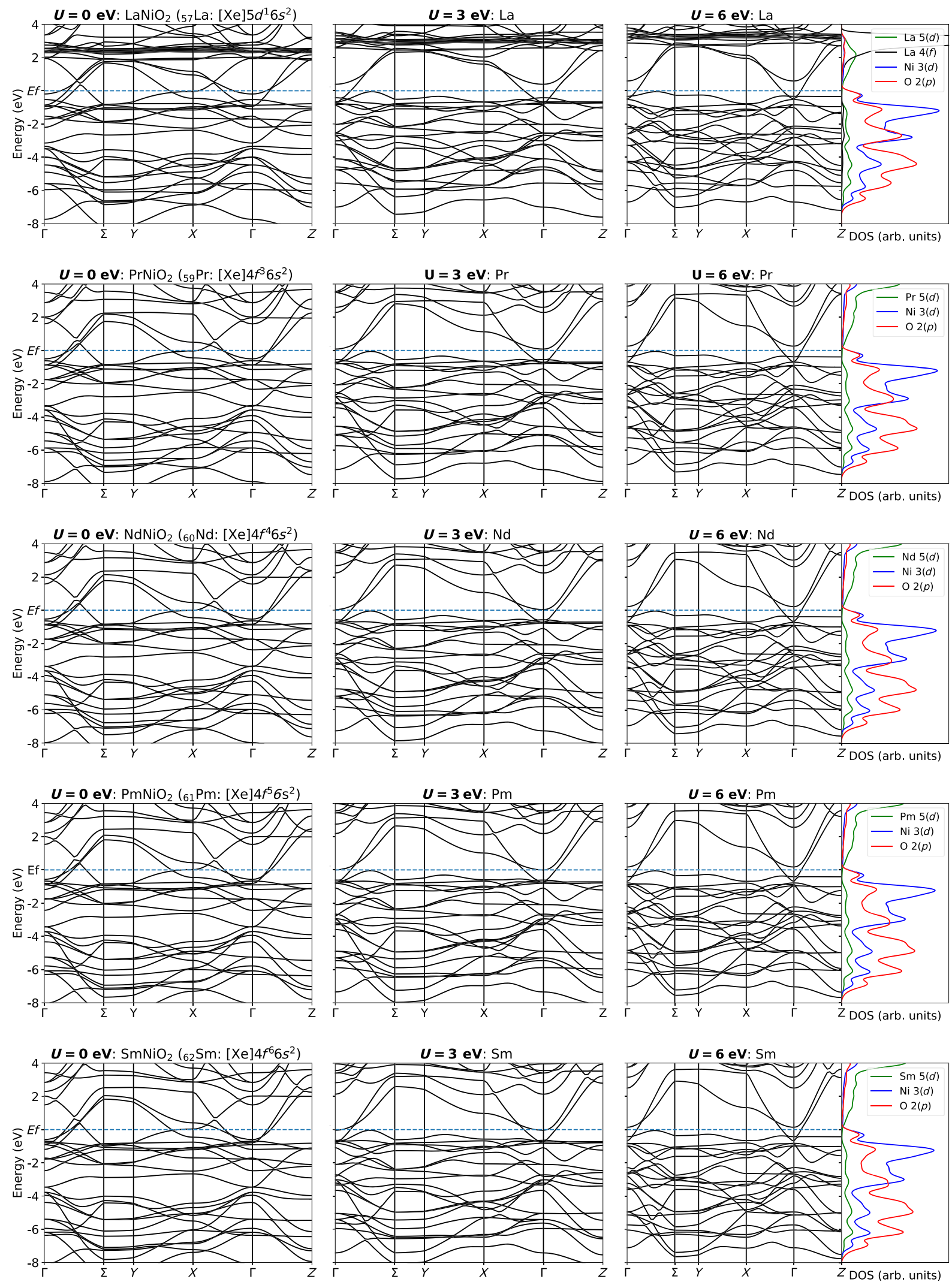

Fig. 6. (Continued). 

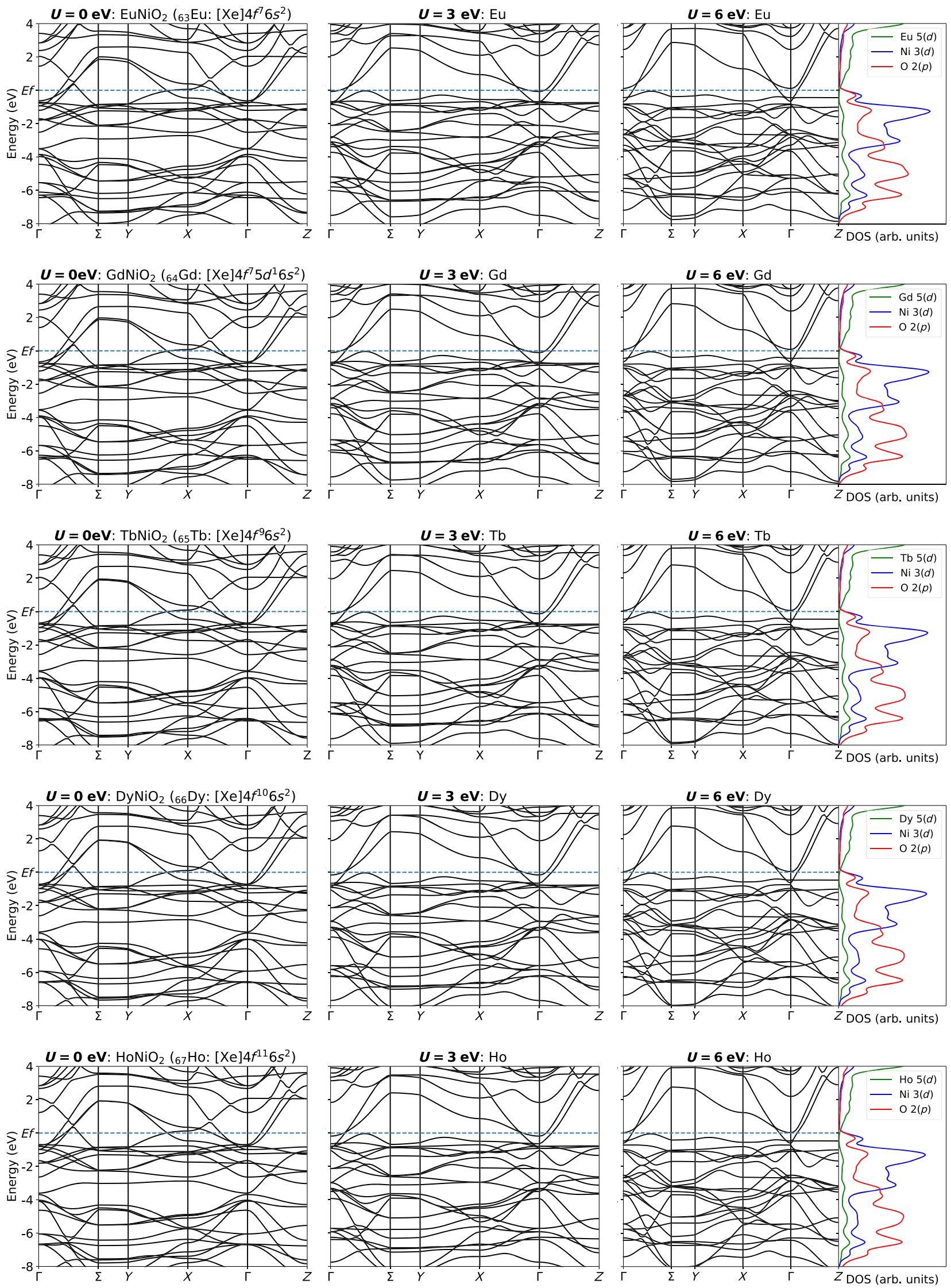

Fig. 6. (Continued). 

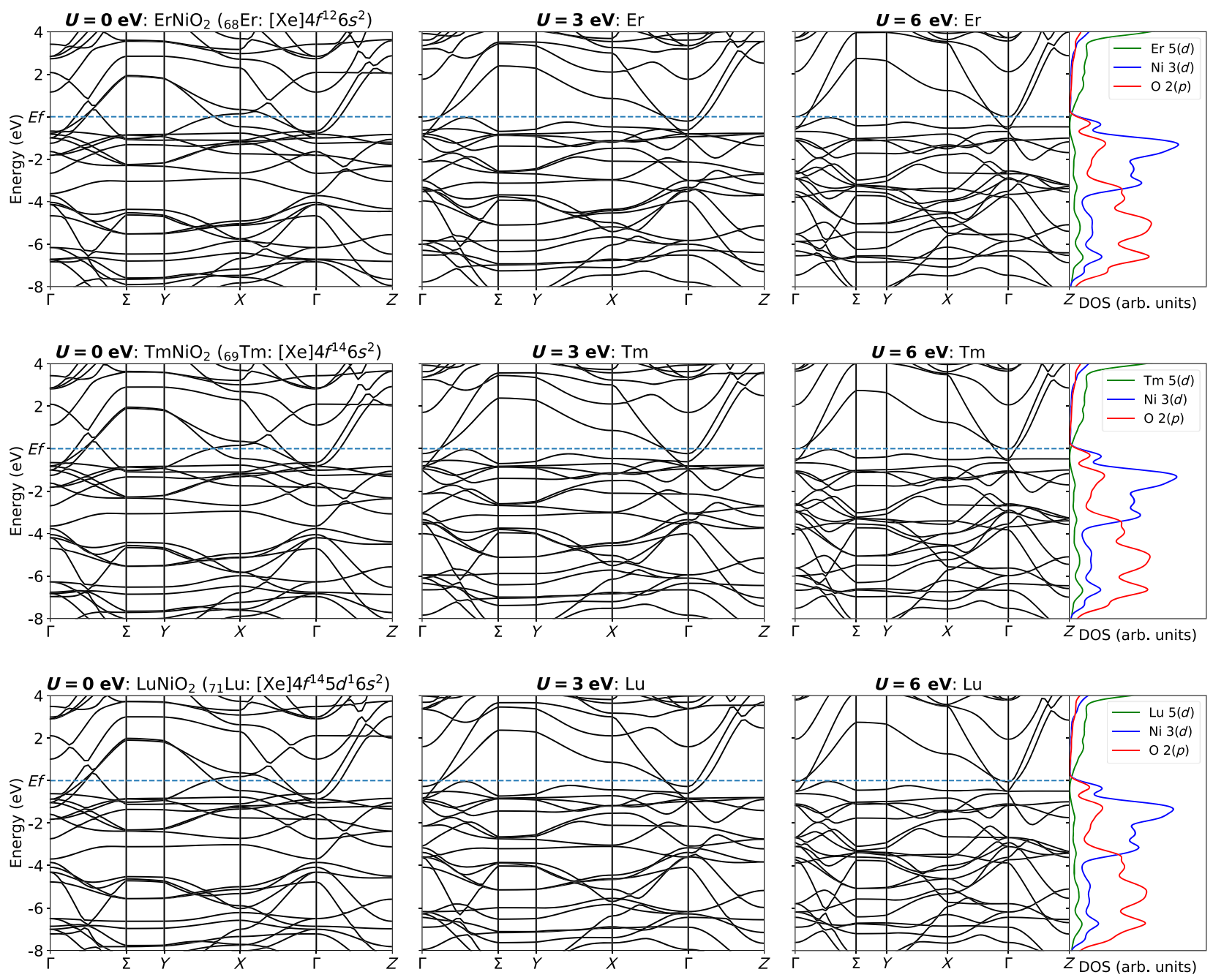

FIG. 6. Band structures of $R \mathrm{NiO}_{2}$ calculated by GGA, GGA $+U(U=3 \mathrm{eV}), \mathrm{GGA}+U(U=6 \mathrm{eV})$, and partial density of states of $R \mathrm{NiO}_{2}$ calculated by $\mathrm{GGA}+U(6 \mathrm{eV})$.

\section{APPENDIX B: $\mathrm{CaCuO}_{2}$ COMPARISON BAND STRUCTURE AND PDOS}
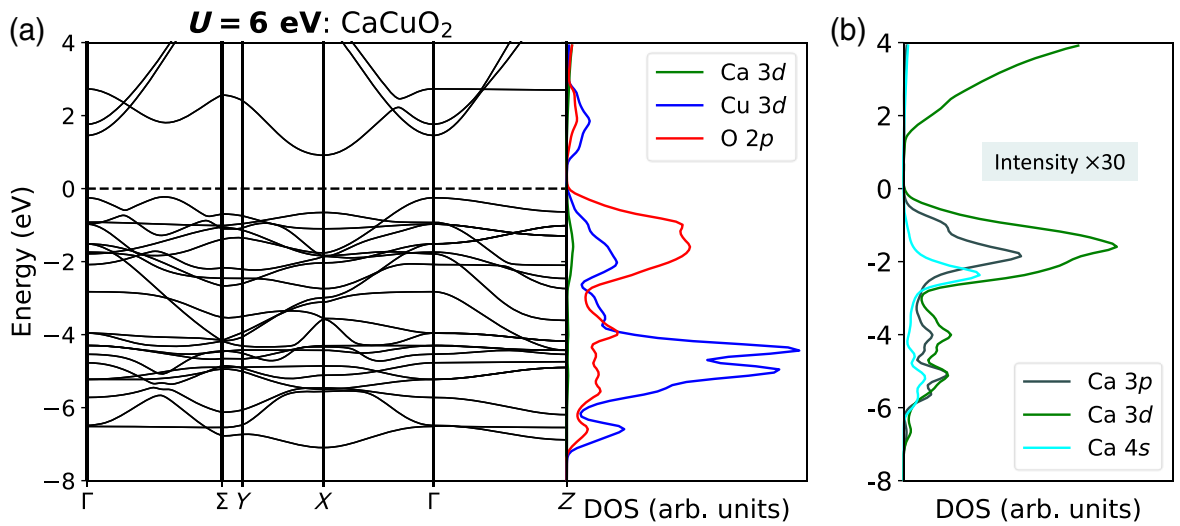

FIG. 7. Electronic structure calculation of $\mathrm{CaCuO}_{2}$. (a) Band structures and partial density of states of $\mathrm{CaCuO}$ calculated by $\mathrm{GGA}+U(U=6 \mathrm{eV})$. (b) Zoom-in intensity of Ca partial density of states of $\mathrm{CaCuO}_{2}$ calculated by $\mathrm{GGA}+U(6 \mathrm{eV})$. 


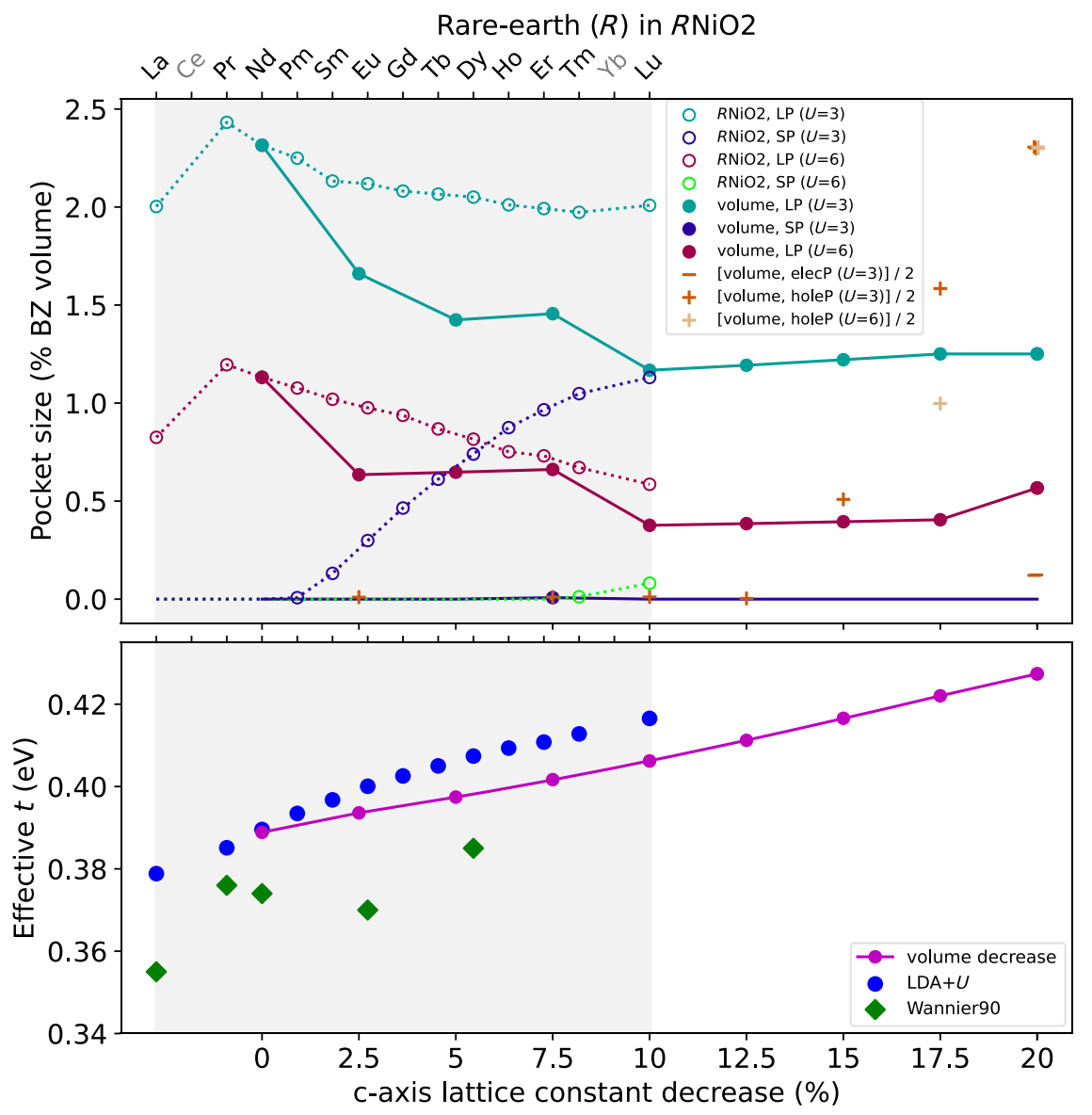

FIG. 8. Comparison between volume change with static $R=\mathrm{Nd}$ and when rare-earth $R$ changes in $R \mathrm{NiO}_{2}$. Top panel: effects on electron pockets for the two scenarios. LP and SP represent the large pocket and small pocket for the $R 5 \mathrm{~d}$ orbital, labeled with circles. When only the volume changes and rare earth stays constant, the second electron pocket (small pocket labeled SP) never substantially appears, and hole pockets start appearing in various parts of the BZ (namely, along $Y-X$ and some along $\Gamma-\Sigma$ ). These anomalous hole pockets (and one electron pocket at 20\%) that appear along the BZ edge are shown at 0.5 times their actual values. Bottom panel: hopping $t$ estimated by $\frac{1}{8} \times\left(\mathrm{Ni3} d_{x^{2}-y^{2}}\right.$ bandwidth) from the noninteracting DFT calculations. Changes in $t$ from exchanging rare-earth $R$ are significantly greater than those from a simple volume change.

\section{APPENDIX C: SIMPLE VOLUME CHANGE WITH STATIC RARE-EARTH ELEMENT $R=$ Nd}

When the volume change calculations were conducted, the relaxed structure of $\mathrm{NdNiO}_{2}$ was used as the equilibrium structure (labeled as $0 \%$ decrease); the $c$-axis lattice constant decreased in $2.5 \%$ increments ranging from $0 \%-20 \% ; a$ - and $b$-axis lattice constants correspondingly decreased by $10 \%$ of the $c$-axis lattice constant's decrease (e.g., when $c$ decreased by $10 \%$, both $a$ and $b$ decreased by $1 \%$ of their equilibrium values). These decreases are comparable to those seen in Fig. 1, where there is a $10 \%$ decrease in the $c$-axis lattice constant and $1 \%$ decrease of the $a$ - and $b$-axis lattice constants going from La to Lu. Although the $10 \%$ decrease in the $c$-axis lattice constant would correspond to the change across the lanthanides, we decided to extend this analysis to $20 \%$ to see the effects of increased "pressure" on the band structures. These volume change calculations are shown in Fig. 8.

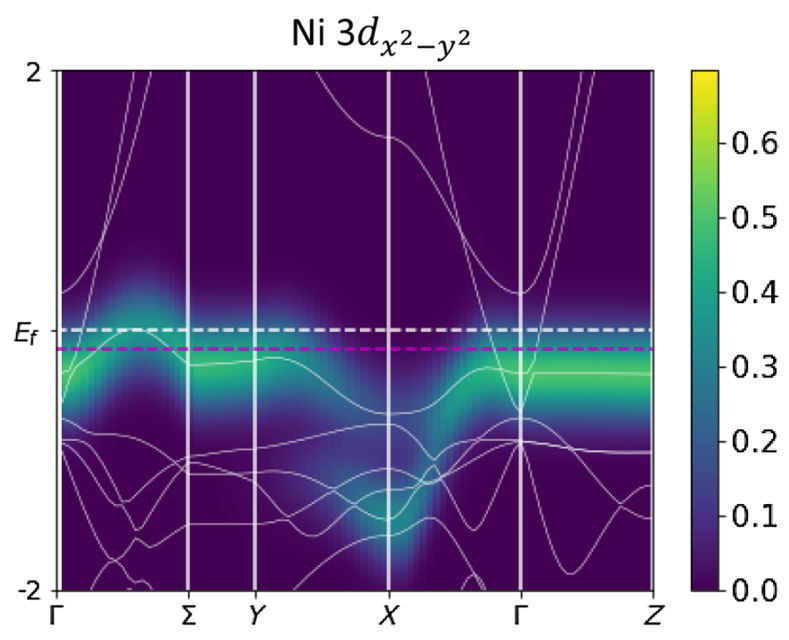

FIG. 9. Estimated Fermi energy shift for $\mathrm{Nd}_{0.8} \mathrm{Sr}_{0.2} \mathrm{NiO}_{2}: E_{f}$ for the parent compound $\mathrm{NdNiO}_{2}$ is marked by the white dashed line, while the estimated $E_{f}$ for $\mathrm{Nd}_{0.8} \mathrm{Sr}_{0.2} \mathrm{NiO}_{2}$ is marked by the red dashed line. 

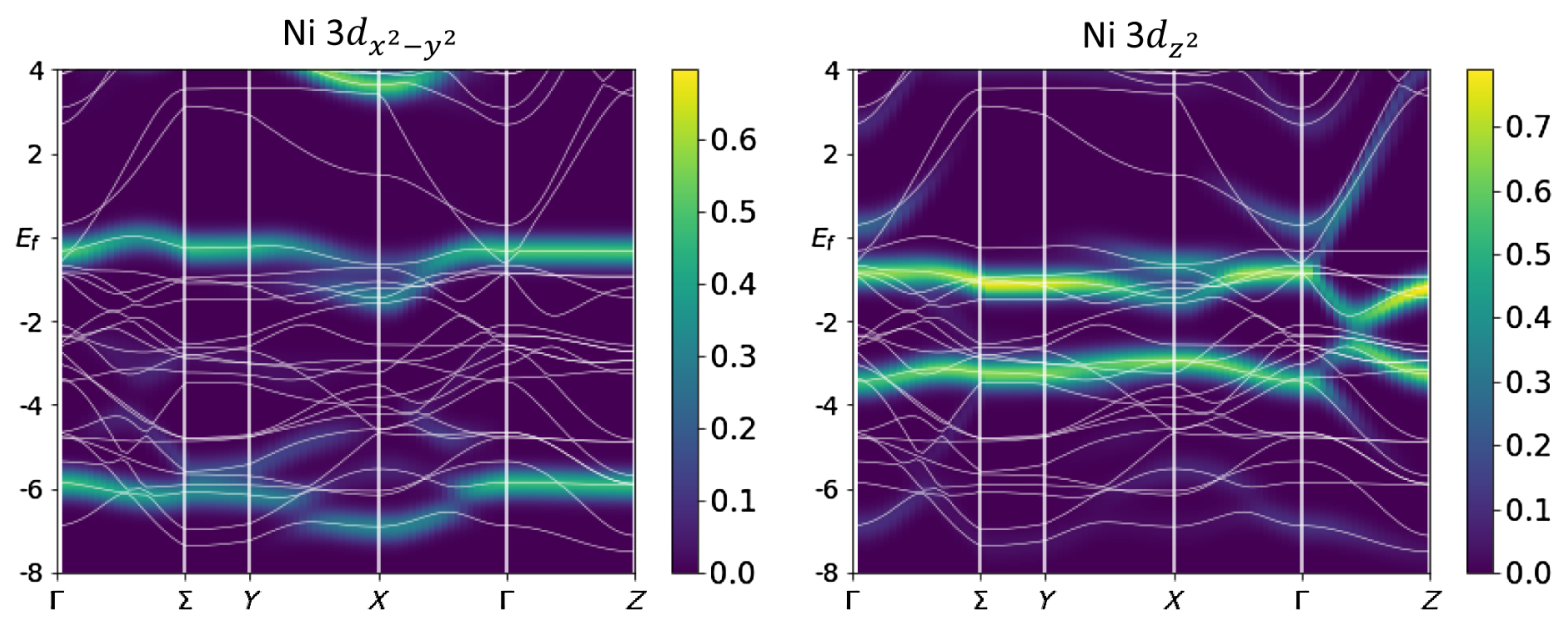

FIG. 10. Highlighted Ni $3 d_{x^{2}-y^{2}}$ and $3 d_{z^{2}}$ orbital character for the $\mathrm{NdNiO}_{2}$ DFT $+U(U=6 \mathrm{eV})$ calculation.

\section{APPENDIX D: RATIONALE TO LEAVE Ni $3 d_{z^{2}}$ OUT OF THE LOW-ENERGY HAMILTONIAN}

Consider the simplistic hypothesis that hole doping applies a rigid shift to the Fermi level and leaves the rest of the band structure unchanged (although we know the Ni-derived bands will behave differently because of correlation effects, we use this simplistic "doping" to get an estimate of which bands could potentially be affected). To approximate how far the Fermi energy will shift, we compare the valence electrons of the parent compound $\mathrm{NdNiO}_{2}$ to the experimentally superconducting compound $\mathrm{Nd}_{0.8} \mathrm{Sr}_{0.2} \mathrm{NiO}_{2}$ [16]. In the $\mathrm{NdNiO}_{2}$ compound, the pseudopotentials include 66 valence electrons in the two-Ni unit cell. However, the total number of valence electrons in the pseudopotentials for $\mathrm{Nd}_{0.8} \mathrm{Sr}_{0.2} \mathrm{NiO}_{2}$ is 65.6. Integrating the density of states for $\mathrm{NdNiO}_{2}$ to this lower filling level gives us a Fermi energy $0.14 \mathrm{eV}$ lower than its current level. In the Fig. 9, this simplified estimate of the Fermi energy for $\mathrm{Nd}_{0.8} \mathrm{Sr}_{0.2} \mathrm{NiO}_{2}$ is shown as the magenta dashed line, and the white dashed line denotes the Fermi energy for $\mathrm{NdNiO}_{2}$ for Ni $3 d_{z^{2}}$ in the $\mathrm{NdNiO}_{2} \mathrm{DFT}+U(U=6 \mathrm{eV})$ calculation. It is evident that even with the simplistic estimate of a rigid shift Fermi level relative to the band structure, all of the relevant information is still contained within the $\mathrm{Ni} 3 d_{x^{2}-y^{2}}$ band since the $3 d_{z^{2}}$ band is far below the "doped" Fermi level. Figure 10 shows the orbitally resolved band structure for a larger energy windows.

[1] J. G. Bednorz and K. A. Müller, Possible High $T_{c}$ Superconductivity in the Ba-La-Cu-O System, Z. Phys. B 64, 189 (1986).

[2] J. Zaanen, G. A. Sawatzky, and J. W. Allen, Band Gaps and Electronic Structure of Transition-Metal Compounds, Phys. Rev. Lett. 55, 418 (1985).

[3] B. Keimer, S. A. Kivelson, M. R. Norman, S. Uchida, and J. Zaanen, From Quantum Matter to High-Temperature
Superconductivity in Copper Oxides, Nature (London) 518, 179 (2015).

[4] J. Bardeen, L. N. Cooper, and J. R. Schrieffer, Theory of Superconductivity, Phys. Rev. 108, 1175 (1957).

[5] D. J. Scalapino, A Common Thread: The Pairing Interaction for Unconventional Superconductors, Rev. Mod. Phys. 84, 1383 (2012).

[6] J. Zaanen and O. Gunnarsson, Charged Magnetic Domain Lines and the Magnetism of High- $T_{c}$ Oxides, Phys. Rev. B 40, 7391 (1989).

[7] J. Zaanen and P. B. Littlewood, Freezing Electronic Correlations by Polaronic Instabilities in Doped $\mathrm{La}_{2} \mathrm{NiO}_{4}$, Phys. Rev. B 50, 7222 (1994).

[8] J. M. Tranquada, D. J. Buttrey, V. Sachan, and J. E. Lorenzo, Simultaneous Ordering of Holes and Spins in $\mathrm{La}_{2} \mathrm{NiO}_{4.125}$, Phys. Rev. Lett. 73, 1003 (1994).

[9] J. M. Tranquada, B. J. Sternlieb, J. D. Axe, Y. Nakamura, and S. Uchida, Evidence for Stripe Correlations of Spins and Holes in Copper Oxide Superconductors, Nature (London) 375, 561 (1995).

[10] R. Comin and A. Damascelli, Resonant X-Ray Scattering Studies of Charge Order in Cuprates, Annu. Rev. Condens. Matter Phys. 7, 369 (2016).

[11] V. I. Anisimov, M. A. Korotin, J. Zaanen, and O. K. Andersen, Spin Bags, Polarons, and Impurity Potentials in $\mathrm{La}_{2-x} \mathrm{Sr}_{x} \mathrm{CuO}_{4}$ from First Principles, Phys. Rev. Lett. 68, 345 (1992).

[12] J. Zhang, D. M. Pajerowski, A. S. Botana, Hong Zheng, L. Harriger, J. Rodriguez-Rivera, J. P. C. Ruff, N. J. Schreiber, B. Wang, Yu.-S. Chen, W. C. Chen, M. R. Norman, S. Rosenkranz, J. F. Mitchell, and D. Phelan, Spin Stripe Order in a Square Planar Trilayer Nickelate, Phys. Rev. Lett. 122, 247201 (2019).

[13] V. Bisogni, S. Catalano, R. J. Green, M. Gibert, R. Scherwitzl, Y. Huang, V. N. Strocov, P. Zubko, S. Balandeh, J.-M. Triscone, G. Sawatzky, and T. Schmitt, Ground-State Oxygen Holes and the Metal-Insulator Transition in the Negative Charge-Transfer Rare-Earth Nickelates, Nat. Commun. 7, 13017 (2016).

[14] M. A. Hayward, M. A. Green, M. J. Rosseinsky, and J. Sloan, Sodium Hydride as a Powerful Reducing Agent for 
Topotactic Oxide Deintercalation: Synthesis and Characterization of the Nickel(I) Oxide $\mathrm{LaNiO}_{2}$, J. Am. Chem. Soc. 121, 8843 (1999).

[15] M. Crespin, P. Levitz, and L. Gatineau, Reduced Forms of $\mathrm{LaNiO}_{3}$ Perovskite. Part 1. Evidence for New Phases: $\mathrm{La}_{2} \mathrm{Ni}_{2} \mathrm{O}_{5}$ and $\mathrm{LaNiO}_{2}$, J. Chem. Soc., Faraday Trans. 2 79, 1181 (1983).

[16] D. Li, K. Lee, B. Y. Wang, M. Osada, S. Crossley, H. R. Lee, Y. Cui, Y. Hikita, and H. Y. Hwang, Superconductivity in an Infinite-Layer Nickelate, Nature (London) 572, 624 (2019).

[17] V. I. Anisimov, D. Bukhvalov, and T. M. Rice, Electronic Structure of Possible Nickelate Analogs to the Cuprates, Phys. Rev. B 59, 7901 (1999).

[18] K.-W. Lee and W. E. Pickett, Infinite-Layer $\mathrm{LaNiO}_{2}: \mathrm{Ni}^{1+}$ Is Not $\mathrm{Cu}^{2+}$, Phys. Rev. B 70, 165109 (2004).

[19] M. Hepting et al., Electronic Structure of the Parent Compound of Superconducting Infinite-Layer Nickelates, Nat. Mater. 19, 381 (2020).

[20] A. S. Botana and M. R. Norman, Similarities and Differences between $\mathrm{LaNiO}_{2}$ and $\mathrm{CaCuO}_{2}$ and Implications for Superconductivity, Phys. Rev. X 10, 011024 (2020).

[21] X. Wu, D. Di Sante, T. Schwemmer, W. Hanke, H. Y. Hwang, S. Raghu, and R. Thomale, Robust $d_{x^{2}-y^{2}}$-Wave Superconductivity of Infinite-Layer Nickelates, Phys. Rev. B 101, 060504(R) (2020).

[22] M. Jiang, M. Berciu, and G. A. Sawatzky, Critical Nature of the Ni Spin State in Doped $\mathrm{NdNiO}_{2}$, Phys. Rev. Lett. 124, 207004 (2020).

[23] L.-H. Hu and C. Wu, Two-Band Model for Magnetism and Superconductivity in Nickelates, Phys. Rev. Research 1, 032046(R) (2019).

[24] Y. Nomura, M. Hirayama, T. Tadano, Y. Yoshimoto, K. Nakamura, and Ryotaro Arita, Formation of a TwoDimensional Single-Component Correlated Electron System and Band Engineering in the Nickelate Superconductor $\mathrm{NdNiO}_{2}$, Phys. Rev. B 100, 205138 (2019).

[25] G.-M. Zhang, Y.-f. Yang, and F.-C. Zhang, Self-Doped Mott Insulator for Parent Compounds of Nickelate Superconductors, Phys. Rev. B 101, 020501(R) (2020).

[26] Y.-H. Zhang and A. Vishwanath, Type-II $t-J$ Model in Superconducting Nickelate $\mathrm{Nd}_{1-x} \mathrm{Sr}_{x} \mathrm{NiO}_{2}$, Phys. Rev. Research 2, 023112 (2020).

[27] P. Werner and S. Hoshino, Nickelate Superconductors: Multiorbital Nature and Spin Freezing, Phys. Rev. B 101, 041104(R) (2020).

[28] F. Bernardini, V. Olevano, and A. Cano, Magnetic Penetration Depth and $T_{c}$ in Superconducting Nickelates, Phys. Rev. Research 2, 013219 (2020).

[29] Y. Gu, S. Zhu, X. Wang, J. Hu, and H. Chen, A Substantial Hybridization between Correlated Ni-d Orbital and Itinerant Electrons in Infinite-Layer Nickelates, Commun. Phys. 3, 84 (2020).

[30] J. Chang, J. Zhao, and Y. Ding, Hund-Heisenberg Model in Superconducting Infinite-Layer Nickelates, Eur. Phys. J. B 93, 220 (2020).

[31] M. Kitatani, L. Si, O. Janson, R. Arita, Z. Zhong, and K. Held, Nickelate Superconductors-A Renaissance of the
One-Band Hubbard Model, npj Quantum Mater. 5, 59 (2020).

[32] M. A. Hayward and M. J. Rosseinsky, Synthesis of the Infinite Layer $\mathrm{Ni}(\mathrm{I})$ Phase $\mathrm{NdNiO}_{2+x}$ by Low Temperature Reduction of $\mathrm{NdNiO}_{3}$ with Sodium Hydride, Solid State Sci. 5, 839 (2003).

[33] P. Giannozzi et al., QUANTUM ESPRESSO: A Modular and Open-Source Software Project for Quantum Simulations of Materials, J. Phys. Condens. Matter 21, 395502 (2009).

[34] A. Al-Zubi, G. Bihlmayer, and S. Blügel, Electronic Structure of Oxygen-Deficient $\mathrm{SrTiO}_{3}$ and $\mathrm{Sr}_{2} \mathrm{TiO}_{4}$, Crystals 9, 580 (2019).

[35] F. H. Spedding, A. H. Daane, and K. W. Herrmann, The Crystal Structures and Lattice Parameters of High-Purity Scandium, Yttrium and the Rare Earth Metals, Acta Crystallogr. 9, 559 (1956).

[36] P. H. Hor, R. L. Meng, Y. Q. Wang, L. Gao, Z. J. Huang, J. Bechtold, K. Forster, and C. W. Chu, Superconductivity above $90 \mathrm{~K}$ in the Square-Planar Compound System $\mathrm{ABa}_{2} \mathrm{Cu}_{3} \mathrm{O}_{6+\mathrm{x}}$ with $A=Y, L a, N d$, Sm, Eu, Gd, Ho, Er and Lu, Phys. Rev. Lett. 58, 1891 (1987).

[37] V. I. Anisimov, J. Zaanen, and O. K. Andersen, Band Theory and Mott Insulators: Hubbard U Instead of Stoner I, Phys. Rev. B 44, 943 (1991).

[38] N. Hamada, Electronic Band Structure of $\mathrm{LaNiO}_{3}$, J. Phys. Chem. Solids 54, 1157 (1993).

[39] V. J. Emery, Theory of High- $T_{\mathrm{c}}$ Superconductivity in Oxides, Phys. Rev. Lett. 58, 2794 (1987).

[40] G. Pizzi et al., Wannier 90 as a Community Code: New Features and Applications, J. Phys. Condens. Matter 32, 165902 (2020).

[41] M. Le Tacon, G. Ghiringhelli, J. Chaloupka, M. M. Sala, V. Hinkov, M. W. Haverkort, M. Minola, M. Bakr, K. J. Zhou, S. Blanco-Canosa, C. Monney, Y. T. Song, G. L. Sun, C. T. Lin, G. M. De Luca, M. Salluzzo, G. Khaliullin, T. Schmitt, L. Braicovich, and B. Keimer, Intense Paramagnon Excitations in a Large Family of High-Temperature Superconductors, Nat. Phys. 7, 725 (2011).

[42] J. M. Tranquada, J. D. Axe, N. Ichikawa, Y. Nakamura, S. Uchida, and B. Nachumi, Neutron-Scattering Study of StripePhase Order of Holes and Spins in $\mathrm{La}_{1.48} \mathrm{Nd}_{0.4} \mathrm{Sr}_{0.12} \mathrm{CuO}_{4}$, Phys. Rev. B 54, 7489 (1996).

[43] G. Aeppli, T. E. Mason, S. M. Hayden, H. A. Mook, and J. Kulda, Nearly Singular Magnetic Fluctuations in the Normal State of a High- $T_{c}$ Cuprate Superconductor, Science 278, 1432 (1997).

[44] M. Kenzelmann, T. Strässle, C. Niedermayer, M. Sigrist, B. Padmanabhan, M. Zolliker, A. D. Bianchi, R. Movshovich, E. D. Bauer, J. L. Sarrao, and J. D. Thompson, Coupled Superconducting and Magnetic Order in $\mathrm{CeCoIn}_{5}$, Science 321, 1652 (2008).

[45] M. A. Ruderman and C. Kittel, Indirect Exchange Coupling of Nuclear Magnetic Moments by Conduction Electrons, Phys. Rev. 96, 99 (1954).

[46] T. Kasuya, A Theory of Metallic Ferro- and Antiferromagnetism on Zener's Model, Prog. Theor. Phys. 16, 45 (1956).

[47] K. Yosida, Magnetic Properties of Cu-Mn Alloys, Phys. Rev. 106, 893 (1957). 
[48] S. Doniach, The Kondo Lattice and Weak Antiferromagnetism, Physica (Amsterdam) 91B+C, 231 (1977).

[49] P. Nozières, Impuretés Magnétiques et Effet Kondo, Ann. Phys. Fr. 10, 19 (1985).

[50] P. Nozières, Some Comments on Kondo Lattices and the Mott Transition, Eur. Phys. J. B 6, 447 (1998).

[51] I. Timrov, N. Marzari, and M. Cococcioni, Hubbard Parameters from Density-Functional Perturbation Theory, Phys. Rev. B 98, 085127 (2018).

[52] M. Le Tacon, G. Ghiringhelli, J. Chaloupka, M. M. Sala, V. Hinkov, M. W. Haverkort, M. Minola, M. Bakr, K. J. Zhou, S. Blanco-Canosa, C. Monney, Y. T. Song, G. L. Sun, C. T. Lin, G. M. De Luca, M. Salluzzo, G. Khaliullin, T. Schmitt, L. Braicovich, and B. Keimer, Intense Paramagnon Excitations in a Large Family of HighTemperature Superconductors, Nat. Phys. 7, 725 (2011).

[53] S. M. Hayden, G. Aeppli, R. Osborn, A. D. Taylor, T. G. Perring, S-W. Cheong, and Z. Fisk, High-Energy Spin Waves in $\mathrm{La}_{2} \mathrm{CuO}_{4}$, Phys. Rev. Lett. 67, 3622 (1991).

[54] S. M. Hayden, G. Aeppli, T. G. Perring, H. A. Mook, and F. Doğan, High-Frequency Spin Waves in $\mathrm{YBa}_{2} \mathrm{Cu}_{3} \mathrm{O}_{6.15}$, Phys. Rev. B 54, R6905 (1996).

[55] L. Braicovich, L. J. P. Ament, V. Bisogni, F. Forte, C. Aruta, G. Balestrino, N. B. Brookes, G. M. De Luca, P. G. Medaglia, F. M. Granozio, M. Radovic, M. Salluzzo, J. van den Brink, and G. Ghiringhelli, Dispersion of Magnetic Excitations in the Cuprate $\mathrm{La}_{2} \mathrm{CuO}_{4}$ and $\mathrm{CaCuO}_{2}$ Compounds Measured Using Resonant X-Ray Scattering, Phys. Rev. Lett. 102, 167401 (2009).
[56] C. P. J. Adolphs, S. Moser, G. A. Sawatzky, and M. Berciu, Non-Zhang-Rice Singlet Character of the First Ionization State of T-CuO, Phys. Rev. Lett. 116, 087002 (2016).

[57] D. Rybicki, M. Jurkutat, S. Reichardt, C. Kapusta, and J. Haase, Perspective on the Phase Diagram of Cuprate HighTemperature Superconductors, Nat. Commun. 7, 11413 (2016).

[58] M. Jurkutat, D. Rybicki, O. P. Sushkov, G. V. M. Williams, A. Erb, and J. Haase, Distribution of Electrons and Holes in Cuprate Superconductors as Determined from ${ }^{17} \mathrm{O}$ and ${ }^{63} \mathrm{Cu}$ Nuclear Magnetic Resonance, Phys. Rev. B 90, 140504(R) (2014).

[59] M. Cococcioni and S. de Gironcoli, Linear Response Approach to the Calculation of the Effective Interaction Parameters in the LDA + U Method, Phys. Rev. B 71, 035105 (2005).

[60] G. Kresse and J. Hafner, Ab Initio Molecular Dynamics for Liquid Metals, Phys. Rev. B 47, 558 (1993).

[61] L. Soderholm, K. Zhang, D. G. Hinks, M. A. Beno, J. D. Jorgensen, C. U. Segre, and I. K. Schuller, Incorporation of Pr in $\mathrm{YBa}_{2} \mathrm{Cu}_{3} \mathrm{O}_{7-\delta}$ : Electronic Effects on Superconductivity, Nature (London) 328, 604 (1987).

[62] C. R. Harris et al., Array Programming with NumPy, Nature (London) 585, 357 (2020).

[63] S. van der Walt, S. C. Colbert, and G. Varoquaux, The NumPy Array: A Structure for Efficient Numerical Computation, Comput. Sci. Eng. 13, 22 (2011).

[64] P. Virtanen et al., SciPy 1.0 Contributors, SciPy 1.0: Fundamental Algorithms for Scientific Computing in Python, Nat. Methods 17, 261 (2020). 